\title{
A new continuous-discontinuous damage model: cohesive cracks via an accurate energy-transfer process
}

\author{
Elena Tamayo-Mas ${ }^{1}$ and Antonio Rodríguez-Ferran ${ }^{1, *}$ \\ ${ }^{1}$ Laboratori de Càlcul Numèric (LaCàN). Departament de Matemàtica Aplicada III \\ Universitat Politècnica de Catalunya, BarcelonaTech, 08034 Barcelona, Spain. \\ e-mail: \{elena.tamayo,antonio.rodriguez-ferran\}@upc.edu, web http://www.lacan.upc.edu
}

\begin{abstract}
SUMMARY
A new continuous-discontinuous strategy to describe failure of quasi-brittle materials is presented. For the early stages of the failure process, a gradient-enhanced model based on smoothed displacements is employed. As soon as the damage parameter exceeds a critical value $D_{\text {crit }}<1$, a cohesive crack is introduced. A new criterion to estimate the energy not yet dissipated by the bulk when switching models from continuous to continuous-discontinuous - is proposed. Then, this energy is transferred to the cohesive crack thus ensuring that the continuous and the continuous-discontinuous strategies are energetically equivalent. Compared to other existing techniques, this new strategy accounts for the different unloading branches of damage models and thus, a more accurate estimation of the energy that has to be transferred is obtained. The performance of this technique is illustrated with one- and two-dimensional examples.
\end{abstract}

Received ...

KEY WORDS: Continuous-discontinuous strategy, regularisation, smoothed displacements, cohesive cracks, energy balance.

\section{INTRODUCTION}

In order to simulate failure of quasi-brittle materials — concrete or rock, for instanceeither continuous or discontinuous approaches may be used. The former are characterised by a continuously differentiable displacement field, while the latter introduce a discontinuous displacement field. That is, discontinuous strategies explicitly model the crack geometry, while in continuous techniques, cracks are represented by continuum regions that have lost their loadcarrying capacity.

On the one hand, continuous models for failure analysis — damage or softening plasticityare used to model the first stages of failure, see [1]. They are based on constitutive laws with strain softening. Thus, if standard local models are used, the prediction of the energy dissipated during the failure process is physically unrealistic, see [2], and the thickness of the damaged zone suffers from pathological sensitivity to the mesh size. This physically unrealistic behaviour can be overcome by means of different approaches, see [3] and the recent review [4]. One of these possible solutions consists of using gradient-type formulations, see [5] and [6], where a partial differential equation - the regularisation equation - is added to the system relating a local state variable and its non-local counterpart. Nevertheless, despite the regularisation, non-local continuous failure models

\footnotetext{
${ }^{*}$ Correspondence to: A. Rodríguez-Ferran, Laboratori de Càlcul Numèric (LaCàN), Universitat Politècnica de Catalunya, BarcelonaTech, 08034 Barcelona, Spain.
} 
cannot describe material separation, which is necessary for many applications. Indeed, modelling the geometry of the crack is relevant in applications such as hydraulic fracture and leakage problems, where the flow of the fluid (and thus, its pressure) strongly depends on the shape and opening of the crack.

On the other hand, discontinuous models incorporate discontinuous displacement fields. In order to characterise them, different approaches, essentially based on the cohesive crack concept, see [7], have been devised. From a numerical viewpoint, the eXtended Finite Element Method (X-FEM) —see [8], [9] and the review [10] - is the most widely used. Nevertheless, these models cannot be employed to simulate the first stages of the failure process, where damage initiation and its diffuse propagation take place.

Recently, some new approaches that merge these two classical theories - damage and fracture mechanics - have been devised, see for instance [11- 17$]$. The main idea of these continuousdiscontinuous strategies is to combine non-local damage mechanics - hence, numerical difficulties associated with local formulations are overcome and physically realistic results of the first stages of failure are obtained- with fracture mechanics, where the displacement field is characterised by means of the X-FEM.

Three relevant issues, when considering the transition - from regularised damage models to evolving cracks-, are (i) the switching criterion, (ii) the crack-path definition, and (iii) the energy consistency. Regarding the first issue, the transition takes place when the damage (the strain or the stress) field reaches a critical damage (strain or stress) value. The definition of this value is relevant: if the switching is carried out when the material is almost fully degraded, traction-free cracks can be introduced; otherwise, cohesive cracks should be inserted. The second issue, that of defining the crack-path, is still a debated issue. Since linear elastic fracture mechanics cannot be used if a regularised bulk is considered, the crack-path cannot be analytically derived and alternative criteria should be used. The third issue consists in how to ensure that the damaged zone is replaced by an energetically equivalent crack. Indeed, if the transition from a continuous to a discontinuous model is carried out when the material is not fully degraded, the energy not yet dissipated by the bulk needs to be transferred to the crack.

Several combined strategies, starting from the pioneering work [11], can be found in the literature. For instance, in [12], embedded discontinuities are incorporated into a non-local damage model once strain reaches a critical value; in [13], traction-free discontinuities are introduced to a softening viscoplasticity model; a similar combined model is proposed in [14], where traction-free cracks are merged with an implicit gradient-enhanced continuum damage model; in [15] energetically equivalent cohesive cracks are introduced once the damage field reaches a critical damage value $D_{\text {crit }}<1$; and similar energetic considerations are made in [16] when dealing with elastic-damage models, in [17] for ductile materials and in [18] for damage-plasticity.

In this paper, a new continuous-discontinuous model is presented, see Figure 11. For the early stages of the failure process, an implicit gradient-enhanced continuum model, where non-locality is added by means of the displacement field, is used. As soon as the damage parameter reaches a critical value $D_{\text {crit }}<1$, a cohesive crack is introduced. That is, the transition - from a purely continuous damage model to a bulk damage model where discontinuities propagate - is triggered when the material is not fully degraded. Thus, a cohesive crack needs to be incorporated. Regarding the definition of the crack-path, here we use the criterion proposed in [19] and the companion paper [20]. In contrast to traditional techniques, where mechanical criteria are used to locate and propagate cracks, here a geometrical approach is employed. More specifically, given a regularised damage field $D(\boldsymbol{x})$, the discontinuity follows the direction dictated by the medial axis of the isoline (or isosurface in $3 \mathrm{D}) D(\boldsymbol{x})=D^{*}$. Special emphasis is placed on the criterion to determine the cohesive law. Here it is defined in such a way that the continuous and the continuous-discontinuous approaches are energetically equivalent. More specifically, a new criterion to determine the fracture energy not yet dissipated in the damaged bulk - so it can be transferred to the cohesive crack - is proposed.

The new continuous-discontinuous approach is presented in Section 2 First, the continuous gradient-type formulation is briefly reviewed in Section 2.1. Then, the coupling between this gradient-enhanced formulation and cohesive cracks is treated in Section 2.2. The proposed criterion 


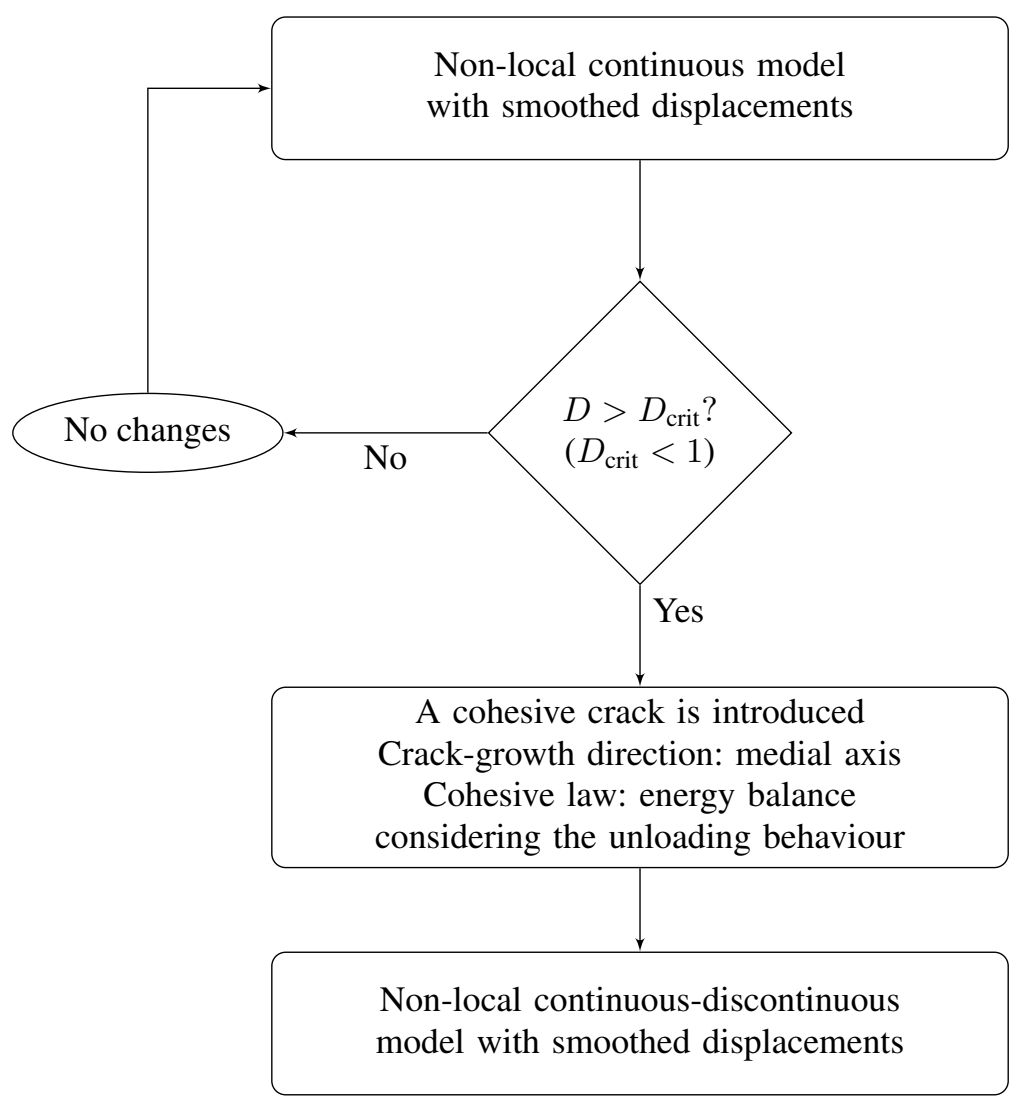

Figure 1. Proposed continuous-discontinuous model.

to estimate the energy not yet dissipated by the bulk when switching models is presented and illustrated by means of some representative numerical examples in Section 3 . The concluding remarks of Section 4 close the paper.

\section{MODEL FORMULATION}

\subsection{Continuous damage model with smoothed displacements}

To describe the early stages of failure, an implicit gradient-enhanced continuum model based on smoothed displacements is used. In this approach, first proposed for damage models in [21] and extended to a general framework in [22], two different displacement fields coexist: (a) the standard or local displacement field $\boldsymbol{u}$ and (b) the gradient-enriched displacement field $\widetilde{\boldsymbol{u}}$. This smoothed displacement field $\widetilde{\boldsymbol{u}}$ drives damage evolution, see Table I. and is the solution of the boundary value problem

$$
\begin{aligned}
\widetilde{\boldsymbol{u}}(\boldsymbol{x})-\ell^{2} \nabla^{2} \widetilde{\boldsymbol{u}}(\boldsymbol{x})=\boldsymbol{u}(\boldsymbol{x}) & \text { in } \Omega \\
\widetilde{\boldsymbol{u}} \cdot \boldsymbol{n}=\boldsymbol{u} \cdot \boldsymbol{n} & \text { on } \partial \Omega \\
\nabla\left(\widetilde{\boldsymbol{u}} \cdot \boldsymbol{t}_{1}\right) \cdot \boldsymbol{n}=\nabla\left(\boldsymbol{u} \cdot \boldsymbol{t}_{1}\right) \cdot \boldsymbol{n} & \text { on } \partial \Omega \\
\nabla\left(\widetilde{\boldsymbol{u}} \cdot \boldsymbol{t}_{2}\right) \cdot \boldsymbol{n}=\nabla\left(\boldsymbol{u} \cdot \boldsymbol{t}_{2}\right) \cdot \boldsymbol{n} & \text { on } \partial \Omega
\end{aligned}
$$

where $\ell$ is the diffusion parameter with dimension of length, $n$ denotes the outward unit normal to $\Omega$ and $\boldsymbol{t}_{1}, \boldsymbol{t}_{2}$ are tangent vectors such that $\left\{\boldsymbol{n}, \boldsymbol{t}_{1}, \boldsymbol{t}_{2}\right\}$ form an orthonormal basis for $\mathbb{R}^{3}$. Note that 
Equations (1b)-(1d) are boundary conditions for the regularisation equation (1a). Therefore, they are prescribed for both the Dirichlet and Neumann boundaries of the mechanical problem.

Table I. Gradient damage model based on smoothed displacements.

$\begin{aligned} \text { Constitutive equation } & \boldsymbol{\sigma}(\boldsymbol{x})=(1-D(\boldsymbol{x})) \boldsymbol{C}: \boldsymbol{\varepsilon}(\boldsymbol{x}) \\ \text { Strains } & \boldsymbol{\varepsilon}(\boldsymbol{x})=\nabla^{s} \boldsymbol{u}(\boldsymbol{x}) \\ \text { Smoothed displacements } & \widetilde{\boldsymbol{u}}(\boldsymbol{x})-\ell^{2} \nabla^{2} \widetilde{\boldsymbol{u}}(\boldsymbol{x})=\boldsymbol{u}(\boldsymbol{x}) \\ \text { Smoothed strains } & \widetilde{\boldsymbol{\varepsilon}}(\boldsymbol{x})=\nabla^{s} \widetilde{\boldsymbol{u}}(\boldsymbol{x}) \\ \text { Smoothed state variable } & Y(\boldsymbol{x})=Y(\widetilde{\boldsymbol{\varepsilon}}(\boldsymbol{x})) \\ \text { Damage evolution } & D(\boldsymbol{x})=D(Y)\end{aligned}$

As detailedly analysed, first in [23] for a two-dimensional setting and then in [20] for 3D problems, these combined boundary conditions satisfy the necessary properties for regularisation - see Table $\Pi$ for a comparison between these required properties and a different set of boundary conditions. Firstly, conditions (1b)-(1d) guarantee reproducibility of order 1 . That is, given a constant strain field $\varepsilon$, the stress field

$$
\boldsymbol{\sigma}(\boldsymbol{x})=(1-D(\widetilde{Y})) \boldsymbol{C}: \boldsymbol{\varepsilon}(\boldsymbol{x})=(1-D(Y)) \boldsymbol{C}: \boldsymbol{\varepsilon}(\boldsymbol{x})
$$

is also constant. Secondly, the relative slip between local and non-local displacements, see conditions (1c) and (1d), allows displacement smoothing along the boundary. This property is especially relevant when dealing with problems where localisation starts at the boundary, as pointed out in [24]. Thirdly, condition (1b) ensures that non-locality vanishes at the boundary in its normal direction, as requested in [25] and [26]. Moreover, combined boundary conditions ensure volume conservation. Indeed, let us suppose constant density and use the divergence theorem. Then,

$$
\int_{\Omega} \nabla \cdot(\widetilde{\boldsymbol{u}}-\boldsymbol{u}) \mathrm{d} \Omega=\int_{\partial \Omega}(\widetilde{\boldsymbol{u}}-\boldsymbol{u}) \cdot \boldsymbol{n} \mathrm{d} \Gamma=0
$$

is satisfied. Note that preservation of volume may be interesting in some constitutive models. For instance, let us assume that the regularised plasticity model presented in [22] is used. Then, preservation of volume ensures that given isochoric local strains $(\nabla \cdot \boldsymbol{u}=0)$, isochoric non-local strains $(\nabla \cdot \widetilde{\boldsymbol{u}}=0)$ are obtained.

\subsection{Continuous-discontinuous damage model with smoothed displacements}

Once the damage parameter $D(\boldsymbol{x})$ reaches a critical value $D_{\text {crit }}$, a crack is introduced in that element. From that moment on, the continuous implicit gradient-enhanced model is coupled with a cohesive crack. In this stage of the process, the bulk $\Omega$ is bounded by $\Gamma=\Gamma_{u} \cup \Gamma_{t} \cup \Gamma_{d}$, as shown in Figure 2(a), Prescribed displacements are imposed on the Dirichlet boundary $\Gamma_{u}$, while tractions are imposed on the Neumann boundary $\Gamma_{t} . \Gamma_{d}$ is the cohesive crack. Its orientation is given by a unit vector $\boldsymbol{n}$ perpendicular to the discontinuity surface. By means of this vector, the two faces of the discontinuity $\Gamma_{d}^{+}$and $\Gamma_{d}^{-}$can be distinguished, see Figure 2(b)

The basic idea of this combined strategy is to describe the two displacement fields by means of the eXtended Finite Element Method (X-FEM). Indeed, and with X-FEM, $\boldsymbol{u}$ and $\widetilde{\boldsymbol{u}}$ can be decomposed as

$$
\begin{aligned}
& \boldsymbol{u}(\boldsymbol{x})=\boldsymbol{u}^{1}(\boldsymbol{x})+\psi(\boldsymbol{x}) \boldsymbol{u}^{2}(\boldsymbol{x}) \\
& \widetilde{\boldsymbol{u}}(\boldsymbol{x})=\widetilde{\boldsymbol{u}}^{1}(\boldsymbol{x})+\psi(\boldsymbol{x}) \widetilde{\boldsymbol{u}}^{2}(\boldsymbol{x})
\end{aligned}
$$


Table II. Summary table: boundary conditions and their properties.

\begin{tabular}{ccccc}
\hline & $\begin{array}{c}\text { Dirichlet } \\
\widetilde{\boldsymbol{u}}=\boldsymbol{u}\end{array}$ & $\begin{array}{c}\text { Nomogeneous } \\
\nabla \widetilde{\boldsymbol{u}} \cdot \boldsymbol{n}=\mathbf{0}\end{array}$ & $\begin{array}{c}\text { Non-homogeneous } \\
\text { Neumann } \\
\nabla \widetilde{\boldsymbol{u}} \cdot \boldsymbol{n}=\nabla \boldsymbol{u} \cdot \boldsymbol{n}\end{array}$ & $\begin{array}{c}\text { Combined } \\
\widetilde{\boldsymbol{u}} \cdot \boldsymbol{n}=\boldsymbol{u} \cdot \boldsymbol{n}\end{array}$ \\
$\begin{array}{c}\nabla\left(\widetilde{\boldsymbol{u}} \cdot \boldsymbol{t}_{1}\right) \cdot \boldsymbol{n}=\nabla\left(\boldsymbol{u} \cdot \boldsymbol{t}_{1}\right) \cdot \boldsymbol{n} \\
\nabla\left(\widetilde{\boldsymbol{u}} \cdot \boldsymbol{t}_{2}\right) \cdot \boldsymbol{n}=\nabla\left(\boldsymbol{u} \cdot \boldsymbol{t}_{2}\right) \cdot \boldsymbol{n}\end{array}$ \\
\hline $\begin{array}{c}\text { Reproducibility } \\
\text { of order 1 }\end{array}$ & $\checkmark$ & $\times$ & $\checkmark$ & $\checkmark$ \\
\hline $\begin{array}{c}\text { Displacement } \\
\text { smoothing } \\
\text { along } \\
\text { the boundary }\end{array}$ & $\times$ & & & $\checkmark$ \\
\hline $\begin{array}{c}\text { Local response } \\
\text { normal } \\
\text { to boundaries }\end{array}$ & $\checkmark$ & $\checkmark$ & $\checkmark$ & $\checkmark$ \\
\hline $\begin{array}{c}\text { Volume } \\
\text { preservation }\end{array}$ & $\checkmark$ & $\times$ & & $\checkmark$ \\
\hline
\end{tabular}

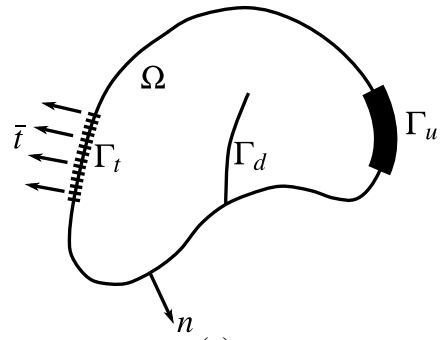

(a)

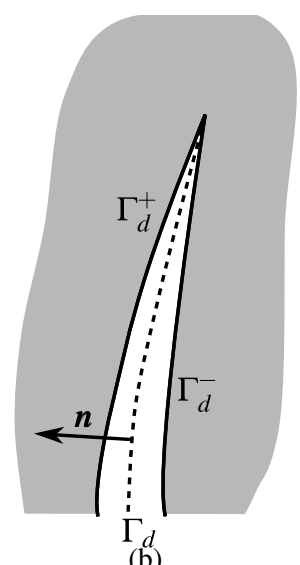

(b)

Figure 2. (a) Notations for a body with a crack subjected to loads and imposed displacements. (b) Notations for the cohesive crack.

where $\boldsymbol{u}^{i}, \widetilde{\boldsymbol{u}}^{i}(i=1,2)$ are continuous fields in $\Omega$ and $\psi$ is the sign function centred at the crack $\Gamma_{d}$ - equals 1 at one side of the discontinuity and equals -1 at the other one. It is noted that if the body $\Omega$ is not entirely crossed by the discontinuity $\Gamma_{d}, \psi$ is ambiguously defined. However, this ambiguity is not relevant, since after the finite element discretisation, the sign function is multiplied by nodal shape functions that vanish in the region where $\psi$ is ambiguous.

2.2.1. Governing equations. The strong form of the equilibrium equation and boundary conditions for the body $\bar{\Omega}=\Omega \cup \Gamma$ without body forces and a cohesive discontinuity $\Gamma_{d}$ is given by

$$
\begin{aligned}
\nabla \cdot \boldsymbol{\sigma} & =\mathbf{0} & & \text { in } \Omega \\
\boldsymbol{\sigma} \cdot \boldsymbol{n} & =\overline{\boldsymbol{t}} & & \text { on } \Gamma_{t} \\
\boldsymbol{\sigma} \cdot \boldsymbol{n} & =\overline{\boldsymbol{t}}_{d} & & \text { on } \Gamma_{d} \\
\boldsymbol{u} & =\boldsymbol{u}^{*} & & \text { on } \Gamma_{u}
\end{aligned}
$$


where $\sigma$ is the Cauchy stress tensor, $\boldsymbol{u}^{*}$ is a prescribed displacement on the Dirichlet boundary, $\overline{\boldsymbol{t}}$ is the traction on the Neumann boundary, $\overline{\boldsymbol{t}}_{d}$ is the traction on the discontinuity surface and $\boldsymbol{n}$ is the outward unit normal to the body. Note that equation $5 \mathrm{5c}$ ) represents traction continuity at the discontinuity $\Gamma_{d}$.

The cohesive tractions are considered to be a function of the crack opening $\llbracket \boldsymbol{u} \rrbracket$, defined as the difference between $\boldsymbol{u}^{+}$and $\boldsymbol{u}^{-}$, where $\boldsymbol{u}^{+}=\boldsymbol{u}_{\Gamma_{d}^{+}}$and $\boldsymbol{u}^{-}=\boldsymbol{u}_{\Gamma_{d}^{-}}$. That is

$$
\llbracket u \rrbracket=\boldsymbol{u}^{+}-\boldsymbol{u}^{-} \quad \text { on } \Gamma_{d}
$$

Different cohesive models can be found in the literature. As reviewed in [4], both initially rigid and initially elastic models can be considered. On the one hand, initially rigid models are based on a monotonic decrease in the cohesive traction, see Figures 3(a) and 3(b) On the other hand, initially elastic models are characterised by an initial positive slope, see Figure 3(c). Here, only initially rigid models are considered, since the cohesive crack is inserted at model switching, when the critical traction $t_{\text {crit }} \neq 0$.

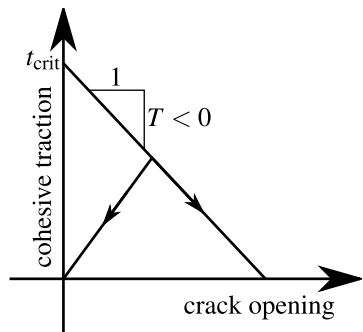

(a)

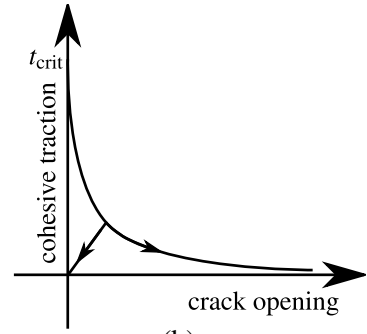

(b)

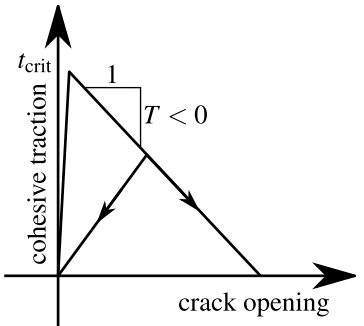

(c)

Figure 3. Typical one-dimensional cohesive models: (a) initially rigid linear cohesive model, (b) initially rigid exponential cohesive model, (c) initially elastic linear cohesive model. Adapted from [4].

Regarding the regularisation of the bulk, smoothed displacements are employed here. Hence, see the discussion of Section 2.1. combined boundary conditions

$$
\begin{aligned}
\widetilde{\boldsymbol{u}}^{i} \cdot \boldsymbol{n} & =\boldsymbol{u}^{i} \cdot \boldsymbol{n} & & \text { on } \partial \Omega \\
\nabla\left(\widetilde{\boldsymbol{u}}^{i} \cdot \boldsymbol{t}_{1}\right) \cdot \boldsymbol{n} & =\nabla\left(\boldsymbol{u}^{i} \cdot \boldsymbol{t}_{1}\right) \cdot \boldsymbol{n} & & \text { on } \partial \Omega \\
\nabla\left(\widetilde{\boldsymbol{u}}^{i} \cdot \boldsymbol{t}_{2}\right) \cdot \boldsymbol{n} & =\nabla\left(\boldsymbol{u}^{i} \cdot \boldsymbol{t}_{2}\right) \cdot \boldsymbol{n} & & \text { on } \partial \Omega
\end{aligned}
$$

where $i=1,2$, are prescribed for the continuous displacement fields $\widetilde{\boldsymbol{u}}^{1}$ and $\widetilde{\boldsymbol{u}}^{2}$. Note that in a discontinuous setting, $\partial \Omega$ is also composed of the crack. Therefore, when the cohesive discontinuity propagates, these boundary conditions change.

2.2.2. Finite element approximation. Regarding the finite element discretisation, local and nonlocal displacements read, in the domain of an element with enhanced nodes, as

$$
\begin{aligned}
& \boldsymbol{u}(\boldsymbol{x}) \simeq \boldsymbol{u}^{h}(\boldsymbol{x})=\mathbf{N}(\boldsymbol{x}) \mathbf{u}^{1}+\psi(\boldsymbol{x}) \mathbf{N}(\boldsymbol{x}) \mathbf{u}^{2} \\
& \widetilde{\boldsymbol{u}}(\boldsymbol{x}) \simeq \widetilde{\boldsymbol{u}}^{h}(\boldsymbol{x})=\mathbf{N}(\boldsymbol{x}) \widetilde{\mathbf{u}}^{1}+\psi(\boldsymbol{x}) \mathbf{N}(\boldsymbol{x}) \widetilde{\mathbf{u}}^{2}
\end{aligned}
$$

where $\mathbf{N}$ is the matrix of standard finite element shape functions, $\mathbf{u}^{1}, \widetilde{\mathbf{u}}^{1}$ are the basic nodal degrees of freedom and $\mathbf{u}^{2}, \widetilde{\mathbf{u}}^{2}$ are the enhanced ones. The discrete format of the equilibrium equation leads to the discrete weak form

$$
\begin{aligned}
\int_{\Omega} \mathbf{B}^{T} \boldsymbol{\sigma} \mathrm{d} \Omega & =\int_{\Gamma_{t}} \mathbf{N}^{T} \overline{\boldsymbol{t}} \mathrm{d} \Gamma \\
\int_{\Omega} \psi \mathbf{B}^{T} \boldsymbol{\sigma} \mathrm{d} \Omega+2 \int_{\Gamma_{d}} \mathbf{N}^{T} \overline{\boldsymbol{t}}_{d} \mathrm{~d} \Gamma & =\int_{\Gamma_{t}} \psi \mathbf{N}^{T} \overline{\boldsymbol{t}} \mathrm{d} \Gamma
\end{aligned}
$$


with $\mathbf{B}$ the matrix of shape function derivatives, while the regularisation equation leads to

$$
\begin{aligned}
\left(\mathbf{M}+\ell^{2} \mathbf{D}\right) \widetilde{\mathbf{u}}^{1}+\left(\mathbf{M}_{\psi}+\ell^{2} \mathbf{D}_{\psi}\right) \widetilde{\mathbf{u}}^{2} & =\left(\mathbf{M}+\ell^{2} \mathbf{K}_{\mathrm{BC}}\right) \widetilde{\mathbf{u}}^{1}+\left(\mathbf{M}_{\psi}+\ell^{2} \mathbf{K}_{\psi, \mathrm{BC}}\right) \mathbf{u}^{2} \\
\left(\mathbf{M}_{\psi}+\ell^{2} \mathbf{D}_{\psi}\right) \widetilde{\mathbf{u}}^{1}+\left(\mathbf{M}+\ell^{2} \mathbf{D}\right) \widetilde{\mathbf{u}}^{2} & =\left(\mathbf{M}_{\psi}+\ell^{2} \mathbf{K}_{\psi, \mathrm{BC}}\right) \mathbf{u}^{1}+\left(\mathbf{M}+\ell^{2} \mathbf{K}_{\mathrm{BC}}\right) \widetilde{\mathbf{u}}^{2}
\end{aligned}
$$

Note that $\mathbf{M}$ and $\mathbf{D}$ are the standard mass and diffusivity matrices already obtained in [21], while $\mathbf{K}_{\mathrm{BC}}$ is the matrix that takes into account the combined boundary conditions. The matrices $\mathbf{M}_{\psi}$, $\mathbf{D}_{\psi}$ and $\mathbf{K}_{\psi, \mathrm{BC}}$ are the enriched mass, diffusivity and boundary matrices, already obtained in the companion paper [20], where traction-free cracks are introduced. Therefore, the only difference between introducing a traction-free crack and a cohesive crack is the second term in the LHS of Equation $9 \mathrm{~b}$. Indeed, if traction-free cracks are introduced, $\overline{\boldsymbol{t}}_{d}=\mathbf{0}$. Nevertheless, if a cohesive crack is considered,

$$
\dot{\overline{\boldsymbol{t}}}_{d}=\boldsymbol{f}(\llbracket \dot{\boldsymbol{u}} \rrbracket)
$$

with $f$ relating the traction rate $\dot{\overline{\boldsymbol{t}}}_{d}$ and the displacement jump rate $\llbracket \dot{\boldsymbol{u}} \rrbracket$. It is noted here that this extra term is multiplied by a factor of two in Equation (9b) because of the sign function $\psi$.

\section{ENERGY BALANCE TO DETERMINE THE COHESIVE LAW}

One important issue concerning the transition from a continuous approach to cohesive cracks is the description of the cohesive law. One means of obtaining the properties of this traction-displacement relation is by enforcing that the energy not yet dissipated by the bulk when switching models is transferred to the cohesive crack. This idea inspired the equivalent crack concept, see [11], and has been used in some combined approaches, see [15], [16] and [27].

The strategy here proposed is based on the same idea. That is, the energy dissipated with a continuous model, $\Psi_{C}$, and with a continuous-discontinuous model, $\Psi_{C D}$, are prescribed to be equal:

$$
\Psi_{C}=\Psi_{C D}
$$

It is noted that, at model switching, the analysis with the continuous model alone is interrupted and replaced by the continuous-discontinuous strategy. Therefore, without a reference continuous simulation, $\Psi_{C}$ is not known and needs to be estimated.

The key idea of our new strategy is the way the energy dissipated by the continuous model $\Psi_{C}$ is computed. For the sake of clarity, this new proposal is first discussed by means of a one-dimensional problem, see Section 3.1. Then, the extension to multidimensional settings is considered, see Section 3.2

\subsection{Energy balance for a uniaxial tension test}

The proposed energy balance is first discussed by means of a uniaxial tension test, see Figure 4(a). The one-dimensional particularisations of the damage model with smoothed displacements, see Table I with $Y(\varepsilon)=\varepsilon$, and the linear softening law

$$
D(Y)=\left\{\begin{array}{cl}
0 & \text { if } \varepsilon<\varepsilon_{0} \\
\frac{\varepsilon_{f}}{\varepsilon_{f}-\varepsilon_{0}}\left(1-\frac{\varepsilon_{0}}{\varepsilon}\right) & \text { if } \varepsilon_{0}<\varepsilon<\varepsilon_{f} \\
1 & \text { if } \varepsilon_{f}<\varepsilon
\end{array}\right.
$$

see Figure 4(b), are used.

A central part of the bar is weakened (10\% reduction in Young's modulus) to trigger localisation. A uniform mesh of 105 elements is considered and the geometric and material parameters are summarised in Table III The numerical tests are displacement-controlled.

Local damage model. To begin with, a continuous strategy with a local damage model is considered. The results are shown in Figure 5. 

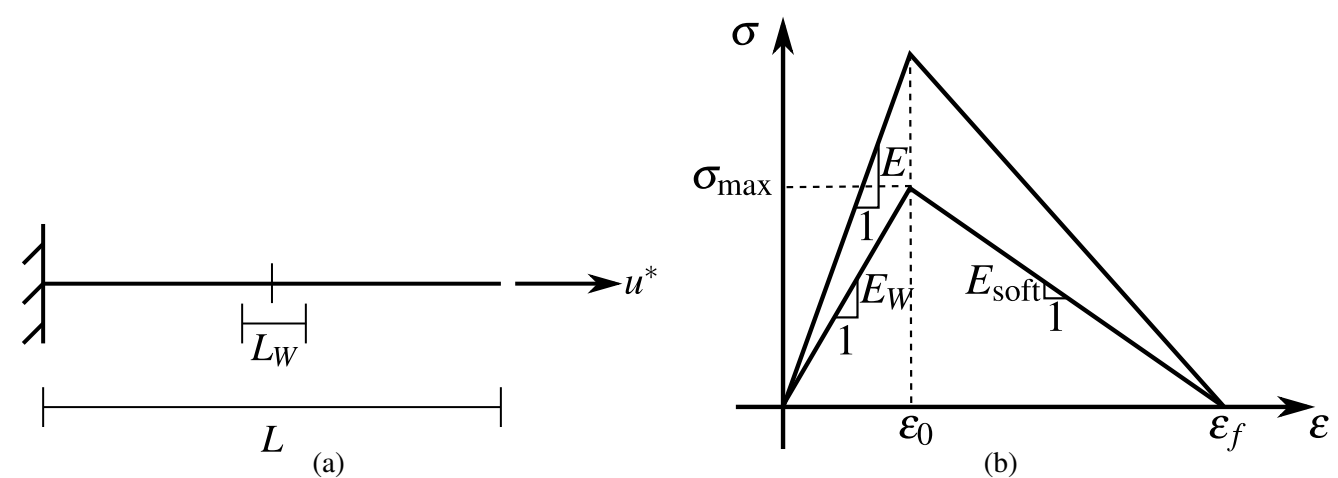

Figure 4. Uniaxial tension test: (a) problem statement; (b) linear softening law.

Table III. Uniaxial tension test: geometrical and material parameters.

\begin{tabular}{lll}
\hline Meaning & Symbol & Value \\
\hline Length of the bar & $L$ & $100 \mathrm{~mm}$ \\
Length of the weaker part & $L_{W}$ & $14 \mathrm{~mm}$ \\
Cross-section of bar & $A$ & $1 \mathrm{~mm}^{2}$ \\
Young's modulus & $E$ & $20000 \mathrm{MPa}$ \\
Young's modulus of the weaker part & $E_{W}$ & $18000 \mathrm{MPa}$ \\
Damage initiation state variable & $\varepsilon_{0}$ & $10^{-4}$ \\
Final state variable & $\varepsilon_{f}$ & $1.25 \times 10^{-2}$ \\
\hline
\end{tabular}

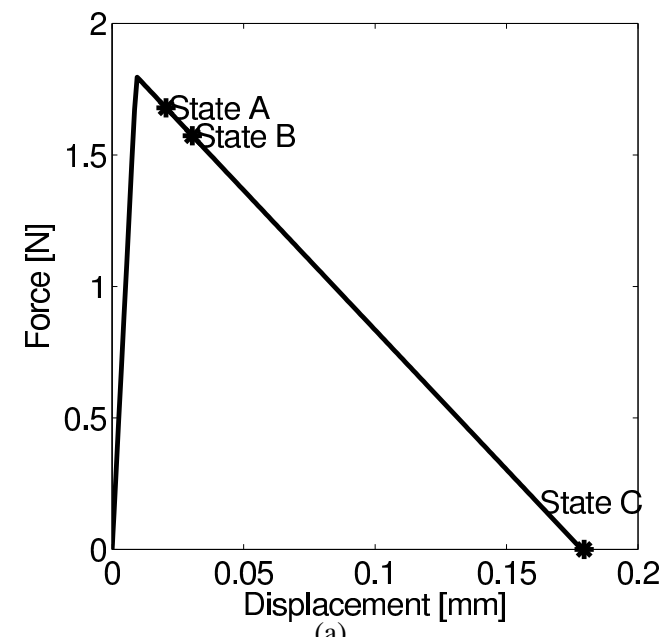

(a)

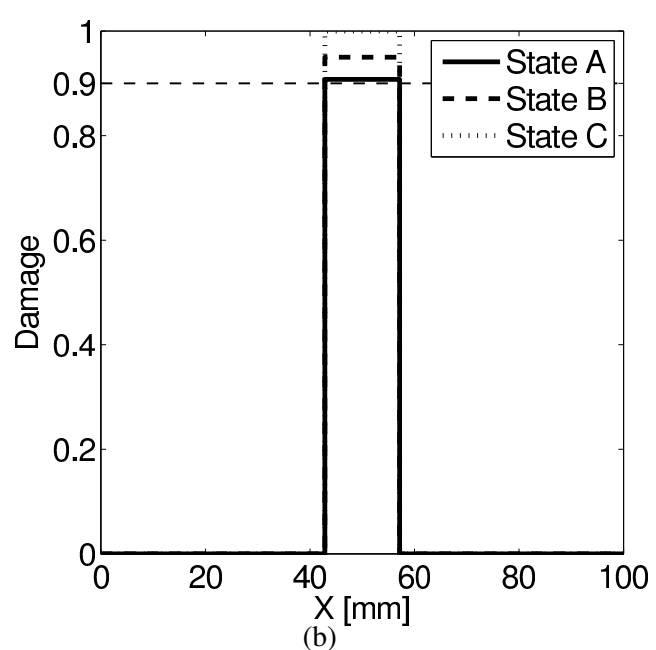

Figure 5. Uniaxial tension test (continuous strategy with a local damage model): (a) force-displacement curves; (b) damage profiles.

On the one hand, Figure 5(a) shows the force-displacement curve. It is noted that it exhibits the two expected branches. Indeed, since in the first load increments the strain is lower than $\varepsilon_{0}$ in all the bar, a first elastic branch with positive slope

$$
\frac{\Delta F}{\Delta u}=\frac{1}{\frac{L-L_{W}}{E}+\frac{L_{W}}{E_{W}}}=196.88 \mathrm{~N} / \mathrm{mm}
$$


is observed. Once the strain reaches the damage initiation threshold in the weakened part, all points in $L_{W}$ unload following the softening branch. Due to equilibrium, the rest of the bar unloads following the elastic branch thus leading to a force-displacement curve with negative slope

$$
\frac{\Delta F}{\Delta u}=\frac{1}{\frac{L-L_{W}}{E}+\frac{L_{W}}{E_{\text {soft }}}}=-10.62 \mathrm{~N} / \mathrm{mm}
$$

where $E_{\text {soft }}=\sigma_{\max } /\left(\varepsilon_{0}-\varepsilon_{f}\right)$, with $\sigma_{\max }=E_{W} \varepsilon_{0}$.

On the other hand, Figure $5(\mathrm{~b})$ shows the damage profiles $D$. Due to locality, the width of the damage profile $\lambda_{D}$ is equal to the length of the weakened part $L_{W}$. In addition, since a continuous strategy is used from the beginning to the end of failure, the damage parameter reaches a maximum value equal to 1 , see the State $\mathrm{C}$ in Figure $5(\mathrm{~b})$.

Let us now consider that as soon as damage reaches a critical value $D_{\text {crit }}=0.9$ (the state A shown in Figure 5], a cohesive crack is introduced at $x=L / 2$ and the proposed continuous-discontinuous strategy is used. This model switching — from the continuous to the combined strategy — entails two main changes.

First, damage is fixed to $D_{\text {crit }}$ in all points in $L_{W}$. Hence, from that moment on, all these points unload following the secant unloading branch with slope $E_{W}\left(1-D_{\text {crit }}\right)$, see Figure 6(a), while the rest of the bar unloads following the elastic branch with slope $E$, see Figure 6(b)
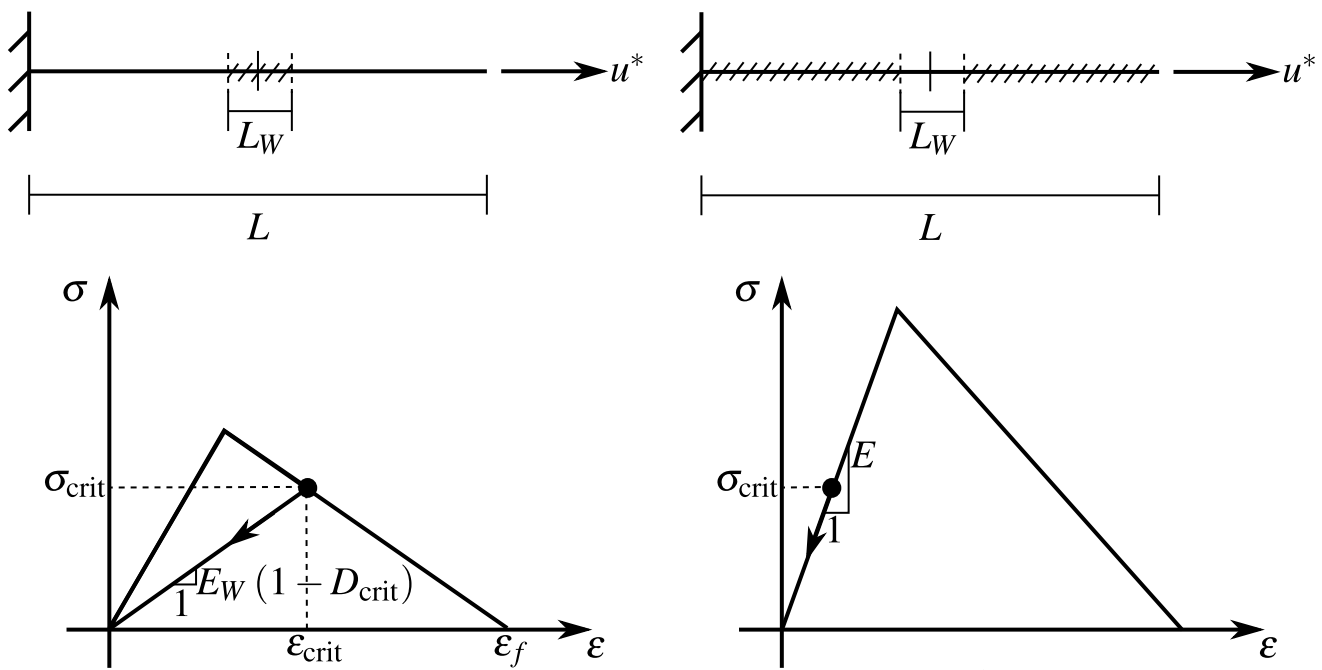

(a)

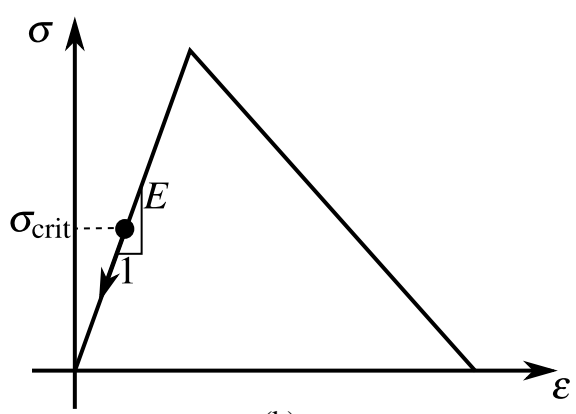

(b)

Figure 6. Once damage reaches a critical value, the model switching is carried out. Hence, (a) points in $L_{W}$ unload following the secant unloading branch with slope $E_{W}\left(1-D_{\text {crit }}\right)$ while (b) the rest of the bar unloads following the elastic branch with slope $E$.

Second, after the switching, no more energy dissipation in the bulk occurs, since all points unload elastically. In other words, the energy dissipated by the bulk if a combined technique is used is the energy already dissipated in the bulk at model switching. Therefore, in order to ensure energy consistency - that is, continuous and continuous-discontinuous strategy should dissipate the same amount of energy - the energy not yet dissipated by the bulk needs to be transferred to the cohesive crack. If a local damage model is employed, this quantity, see Figure 7(a), can be exactly computed. Indeed, for each point of the bar, the energy not yet dissipated is (at model switching) a known quantity. First, due to the elastic response, outside the damaged zone, this quantity is

$$
\psi_{C}^{\text {elas }}=0
$$

Second, for each point in $L_{W}$, the energy not yet dissipated is equal to

$$
\psi_{C}^{\mathrm{dam}}=\frac{1}{2} \sigma_{\mathrm{crit}} \varepsilon_{f},
$$


see Figure 7(b), Therefore the total amount of energy that needs to be transferred to the cohesive crack is

$$
\Psi_{\text {transfer }}=A L_{W} \frac{1}{2} \sigma_{\text {crit }} \varepsilon_{f} .
$$

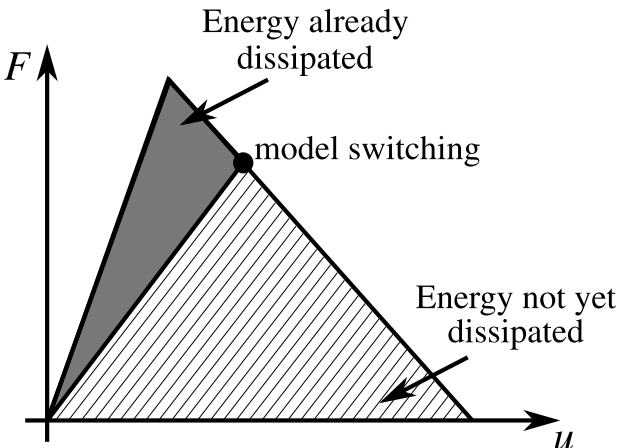

(a)

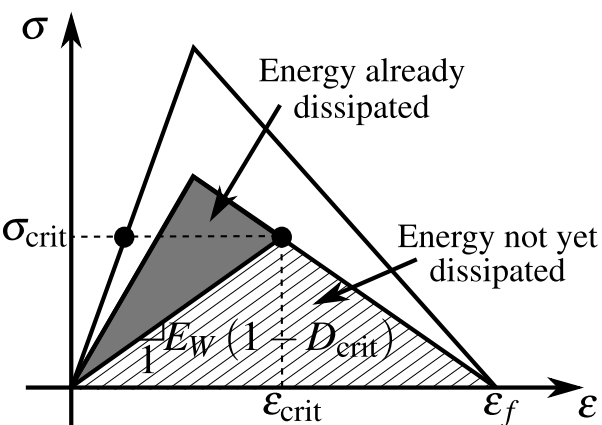

(b)

Figure 7. (a) The energy that needs to be transferred to the crack (striped area) can be exactly computed due to the local behaviour of the solution. (b) Outside the damaged zone, this quantity is 0 , while for each point inside $L_{W}$, this quantity is $\sigma_{\text {crit }} \varepsilon_{f} / 2$

Since the unloading behaviour of the damage model is linear, it is natural to enforce the same behaviour for the cohesive law. Therefore, a linear traction-separation law with slope $T$ is considered, see Figure 8 Then, the exact value of $T$ is obtained by prescribing that the energy not yet dissipated by the bulk at model switching, see Equation (18), is transferred to the crack. Thus,

$$
T=-\frac{\sigma_{\text {crit }}}{A L_{W} \varepsilon_{f}}=-9.40 \mathrm{~N} / \mathrm{mm}^{3}
$$

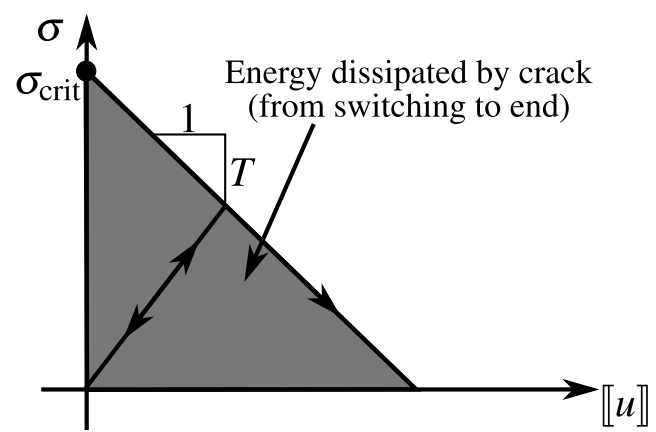

Figure 8. If a linear traction-separation law is considered, the energy dissipated by the crack (area under the

$$
\sigma-\llbracket u \rrbracket \text { curve }) \text { is }-\sigma_{\text {crit }}^{2} /(2 T)
$$

The results for the continuous and the combined strategies are shown in Figure 9. As shown in Figure 9(a) the two strategies are energetically equivalent. Indeed, the force-displacement curve obtained with the combined strategy overlaps the curve obtained with the continuous approach. The difference between these two strategies can be seen in Figure 9(b). On the one hand, if a continuous strategy is employed, the damage profile reaches a maximum value equal to 1 . On the other hand, if a combined strategy is used, damage is fixed to $D_{\text {crit }}=0.9$ in all points of $L_{W}$. That is, after switching, all energy dissipation is due to crack opening and there is no more energy dissipation due to bulk degradation. 


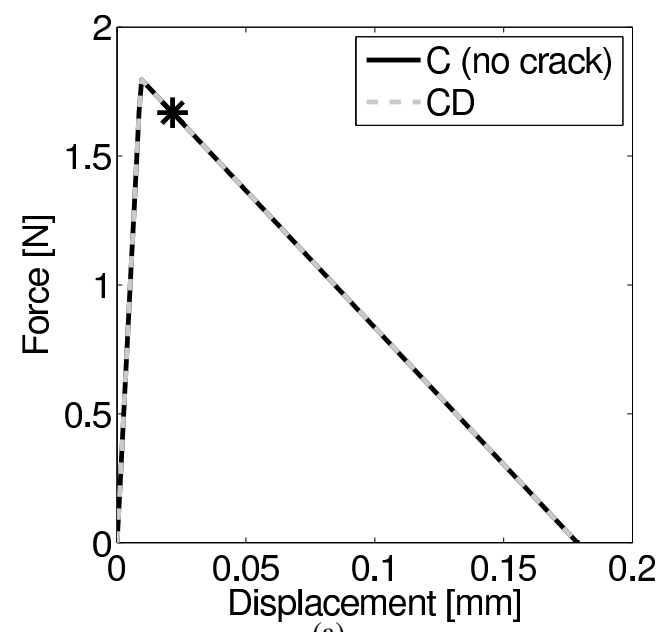

(a)

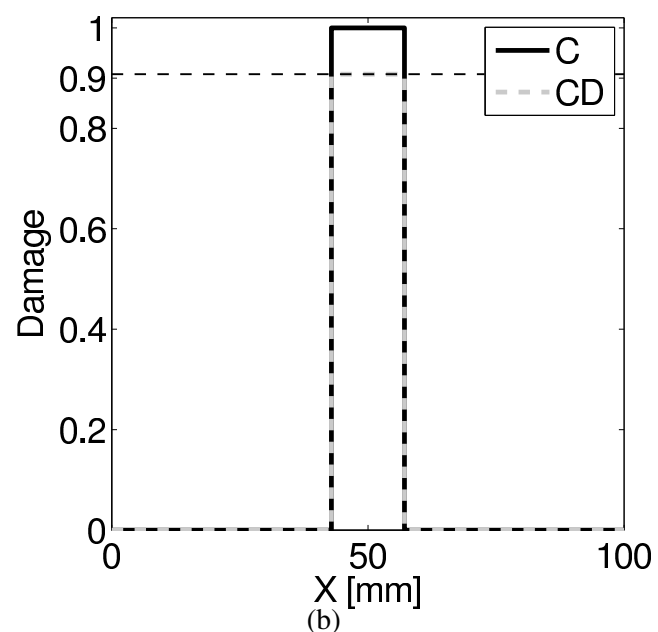

Figure 9. Uniaxial tension test (continuous and continuous-discontinuous approaches with a local damage model): (a) force-displacement curves; (b) damage profiles.

Non-local damage model. The uniaxial tension test is simulated now with a non-local damage model. A characteristic length $\ell=\sqrt{5} \mathrm{~mm}$ is chosen. First, the continuous strategy is employed. Results are shown in Figure 10.

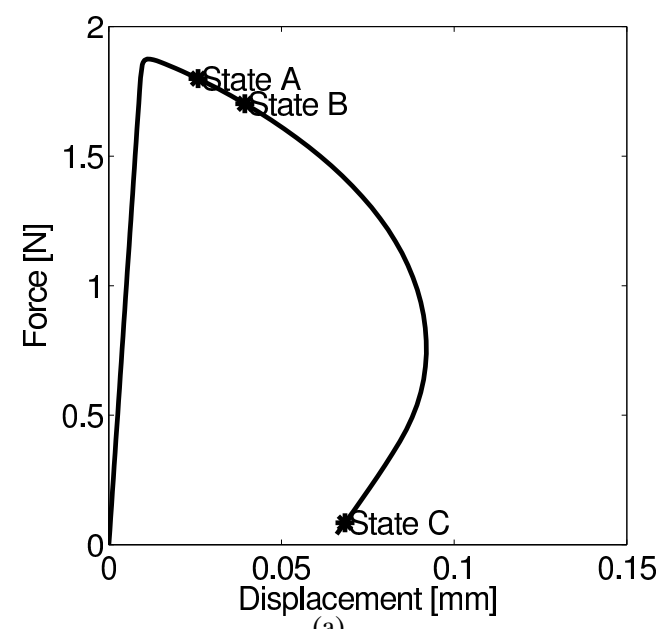

(a)

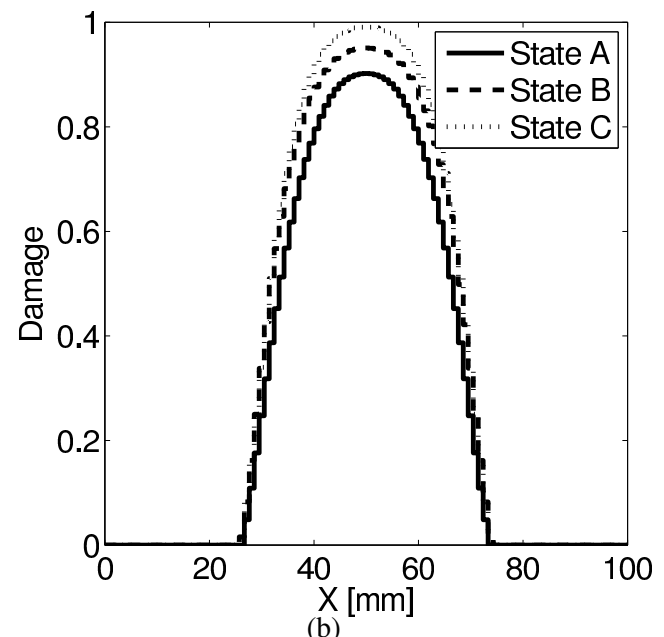

(b)

Figure 10. Uniaxial tension test (continuous strategy with a non-local damage model): (a) forcedisplacement curves; (b) damage profiles.

Figure 10(a) shows the force-displacement curve. Analogously to local results, a first elastic branch, whose slope is given by Equation (14), is observed. Nevertheless, due to non-locality, the force-displacement behaviour after the peak force is reached is qualitatively different. If a local model is used, all points in $L_{W}$ reach the damage initiation strain $\varepsilon_{0}$ at the same time and all points start to unload following the softening branch simultaneously. Thus, the stiffness of the bar is piecewise constant: $E, E_{\text {soft }}, E$. However, if a non-local model is employed, the non-homogeneous behaviour leads to a stiffness that is not piecewise constant.

Damage profiles are shown in Figure 10(b) Analogously to local results, see Figure 5(b), if a continuous strategy is employed from the beginning to the end of failure, the damage parameter reaches a maximum value equal to 1 , see the State $C$ in Figure 10(b) Nevertheless, compared to 
local damage results, two main differences arise. First, the damage profile is not piecewise constant. Second, the width of damage profile $\lambda_{D}$ is not equal to the length of the weakened part. In fact, it depends on the maximum between the width of the localisation zone $\lambda_{\text {loc }}$ (that depends on the characteristic length $\ell$ ) and $L_{W}$, see [22] for a detailed localisation analysis that provides an approximation to $\lambda_{\mathrm{loc}}$. If the proposed parameters are used $(\ell=\sqrt{5} \mathrm{~mm}$ and the parameters of Table III), $\lambda_{\text {loc }}>L_{W}$ and thus, the damaging zone depends on $\ell$ (rather than $L_{W}$ ). These two key differences result from non-locality.

As discussed for the local model, let us consider now that as soon as damage reaches a critical value $D_{\text {crit }}=0.9$ (the state A shown in Figure 10), a cohesive crack (with a linear traction-separation law, see Figure 8) is introduced at $x=L / 2$ and the proposed continuous-discontinuous strategy is used. As discussed above, this model switching has two main consequences.

First, due to the cohesive law, all points in $\lambda_{D}$ unload following the secant unloading branch with slope

$$
E(x)(1-D(x))=\left\{\begin{array}{cc}
E_{W}(1-D(x)) & \text { if } x \in L_{W} \\
E(1-D(x)) & \text { otherwise }
\end{array}\right.
$$

Note that, compared to the local framework, only the point located at $x=L / 2$, see Figure 11(b), unloads following the branch with slope $E_{W}\left(1-D_{\text {crit }}\right)$. Indeed, the rest of the points in $\lambda_{D}$ unload following the secant unloading branch with the stiffness at model switching, see Figure 11(a) while the rest of the bar unloads following the elastic branch with slope $E$, see Figure 11(c)

Second, analogously to the local model, no more energy dissipation in the bulk occurs after switching strategies. Therefore, in order to ensure energy consistency, the energy not yet dissipated by the bulk needs to be transferred to the cohesive crack. If a non-local damage model is employed, this quantity, see Figure 12(a), cannot be exactly computed (without a reference continuous simulation). Indeed, for each point of the bar, the energy not yet dissipated depends on the unloading branch, which is not known at model switching, see Figure 12(b). Therefore, it needs to be estimated as accurately as possible.

One possible way to estimate this energy consists of assuming that all points in $\lambda_{D}$ unload following the local softening branch (from switching to zero stress). This assumption, shown schematically in Figure 13 and made in [15], is quite crude if a non-local model is used: due to non-locality, only the point $x=L / 2$ unloads following this softening branch, while all the other points in $\lambda_{D}$ unload secantly to the origin. Hence, this leads to an overestimation of the energy to be transferred to the cohesive crack.

A more accurate estimation may be obtained if the unloading behaviour - either secant or softening - is taken into account. The key idea of our method is to estimate the energy to be transferred by means of the tangent line to $\sigma(\varepsilon)$ at model switching, see Figure 14. In other words, we propose to estimate this energy by computing the numerical derivative $\mathrm{d} \sigma(\varepsilon) / \mathrm{d} \varepsilon$ at switching. Here, the difference derivative

$$
\frac{\mathrm{d} \sigma}{\mathrm{d} \varepsilon}\left(\varepsilon^{n}\right) \simeq \frac{\sigma\left(\varepsilon^{n}\right)-\sigma\left(\varepsilon^{n-1}\right)}{\varepsilon^{n}-\varepsilon^{n-1}}
$$

is used, where $\varepsilon^{n}$ and $\varepsilon^{n-1}$ stand for the strain at current time-step $t^{n}$ and at previous time-step $t^{n-1}$ respectively.

It must be stressed that, for simplicity, an abuse of notation is being made. Indeed, due to nonlocality, the stress $\sigma$ does not depend only on the strain but also on the smoothed strain $\widetilde{\varepsilon}$, see Table If for details. Thus, the stress $\sigma(\varepsilon)$ is used here to indicate $\sigma(\varepsilon, \widetilde{\varepsilon})=\sigma(\varepsilon, \varepsilon(\widetilde{\varepsilon}))$.

It is noted that a better approximation of the energy to be transferred may be obtained if, after switching models, some load increments with the continuous approach are carried out, see Figure 15. Indeed, once the model switching is determined, some extra load steps with the continuous model can be carried out to estimate the energy not yet dissipated by the bulk with more accuracy. Then, back to the switching point, the simulation is resumed with the continuous-discontinuous strategy. The computational cost of this refinement is marginal, because only a few load steps are 


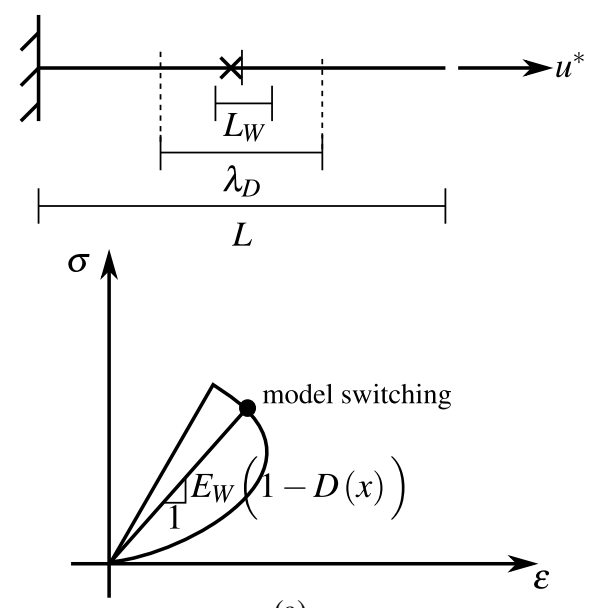

(a)
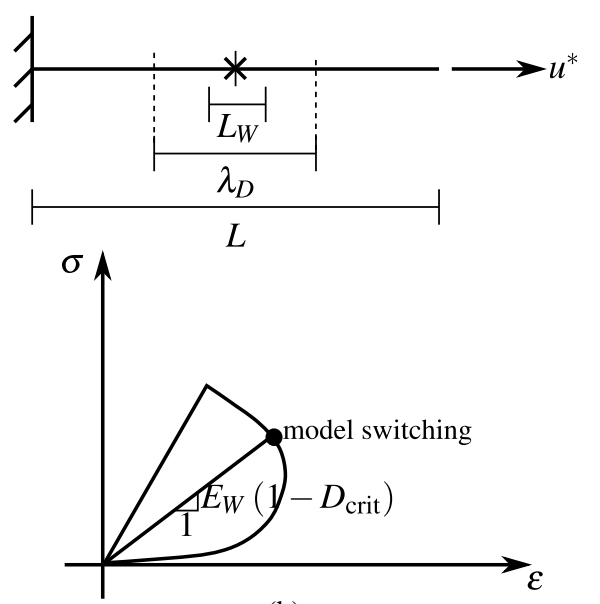

(b)

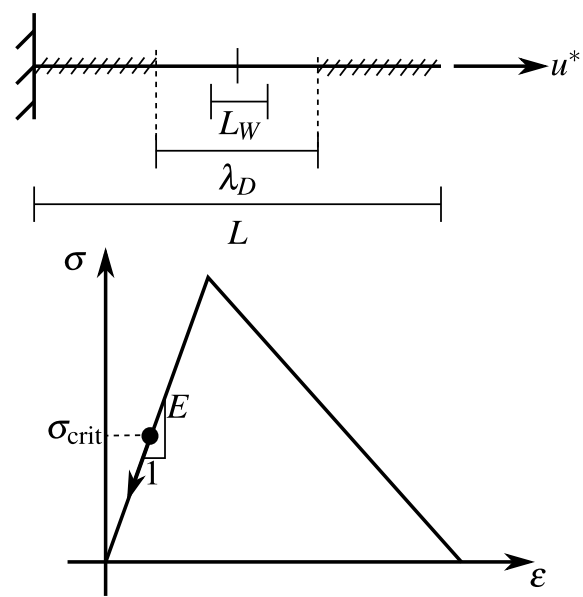

(c)

Figure 11. Once damage reaches a critical value, the model switching is carried out. Hence, (a) points in $\lambda_{D}$ unload following the secant unloading branch with slope $E(x)(1-D(x))$. In contrast to local models, (b) here only the point $x=L / 2$ unloads following the branch with slope $E_{W}\left(1-D_{\text {crit }}\right)$. (c) All points outside the damaged zone $\lambda_{D}$ unload following the elastic branch with slope $E$.

computed twice (first with the continuous approach and then with the continuous-discontinuous one).

The results for the combined strategy (with the cohesive slope obtained by prescribing the proposed energy balance) are shown in Figure 16. Both the continuous and continuousdiscontinuous results are plotted.

In Figure 16(b), the profiles $D$ are shown. As discussed for the local model, the damage profile reaches a maximum value equal to 1 if a continuous strategy is used. If a combined technique is employed, damage is fixed to $D_{\text {crit }}$ at $x=L / 2$ and, after switching, there is no more energy dissipation due to bulk deformation.

Figure 16(a) shows the force-displacement curves. Here, the cohesive parameter $T$ computed by means of the tangent line to $\sigma(\varepsilon)$ at switching point is used. As seen, the area under the combined force-displacement curve (Area $=2.88 \mathrm{~mJ}$ ) is larger than twice the area under the continuous curve $($ Area $=1.29 \mathrm{~mJ})$. This is due to the fact that the energy to be transferred to the cohesive crack has been overestimated. In order to obtain a better approximation of this energy, and suggested by the above discussion, some extra load steps with the continuous technique are carried out. Thus, a 


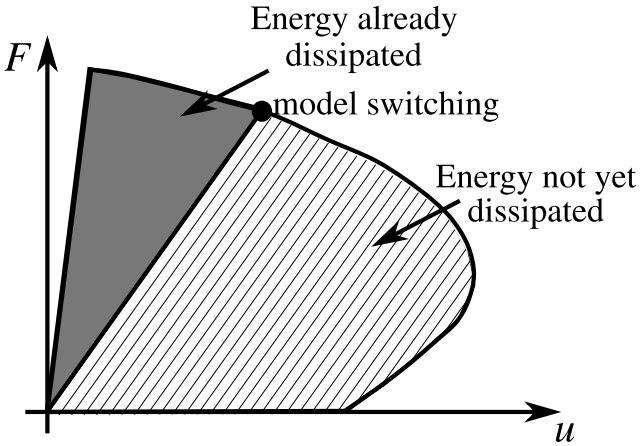

(a)

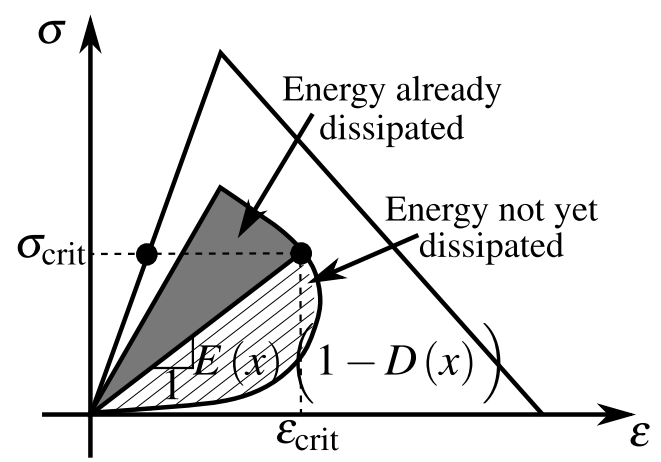

(b)

Figure 12. (a) In contrast to local models, the energy that needs to be transferred to the crack (the energy not yet dissipated by the bulk at model switching) cannot be exactly computed, since (b) for each point in $\lambda_{D}$, the energy not yet dissipated depends on an unloading behaviour, which is not known at model switching.
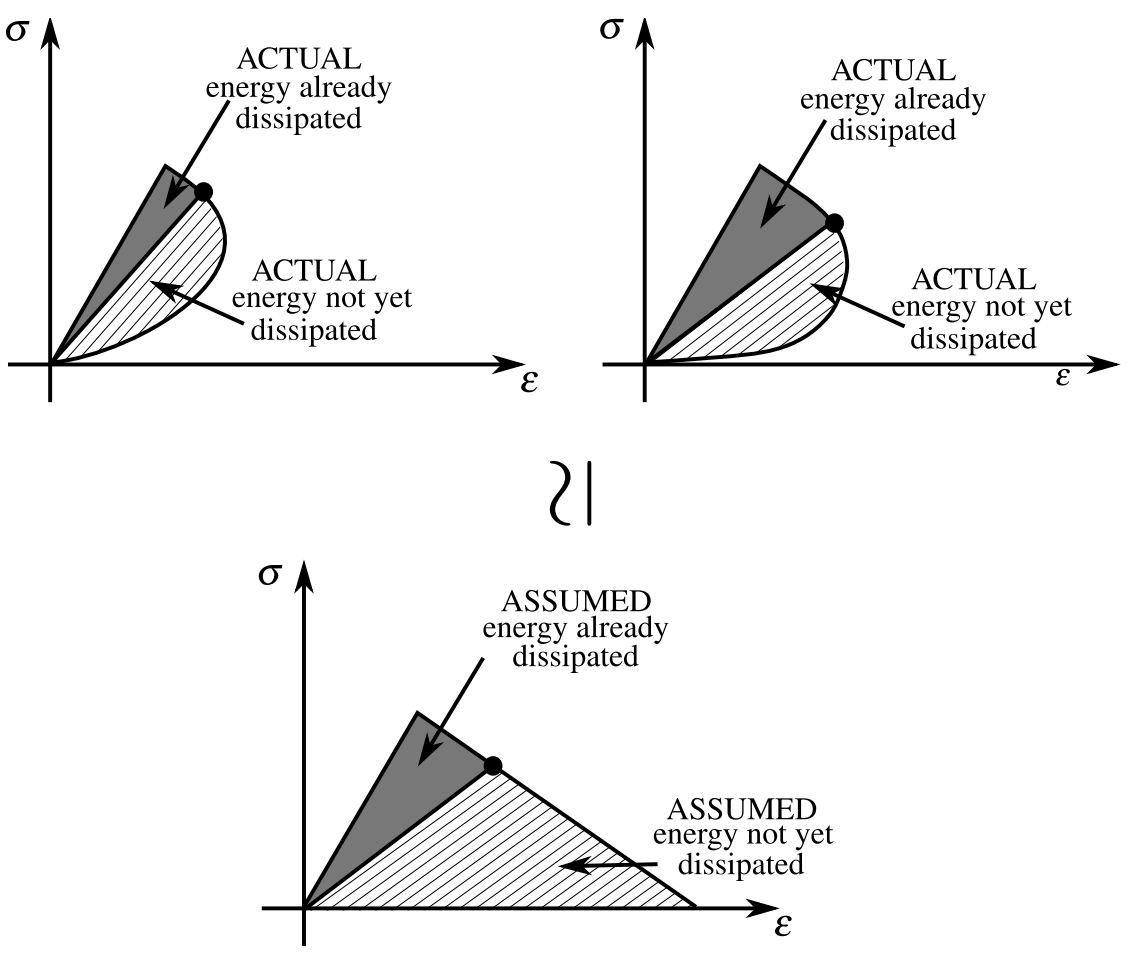

Figure 13. If all points in $\lambda_{D}$ are considered to unload following the softening branch, the energy to be transferred is overestimated.

better solution, see Figure 17(a), is obtained. It is observed that the more extra steps we use, the more accurate the energy to be transferred is, see Figure 17(b)

It is noted that the linear behaviour of the force-displacement curve obtained with the combined approach is due to two reasons. First, a linear cohesive law is considered. Second, once the crack is introduced, the stiffness of the bar is constant. Indeed, since damage is frozen in all the damaged bulk, each point of the damaged band unloads with a constant elastic stiffness $E(x) \cdot(1-D(x))$. 


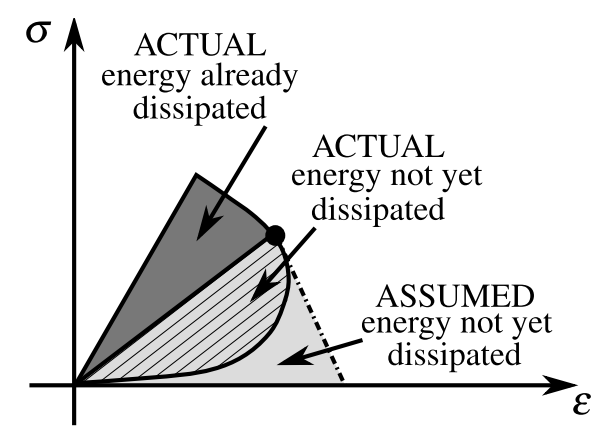

Figure 14. For a given point in $\lambda_{D}$, the energy not yet dissipated by the bulk (striped area) is estimated with the tangent line to $\sigma(\varepsilon)$ (dash-dot line). Hence, an approximation (light grey area) of the actual remaining energy is computed.

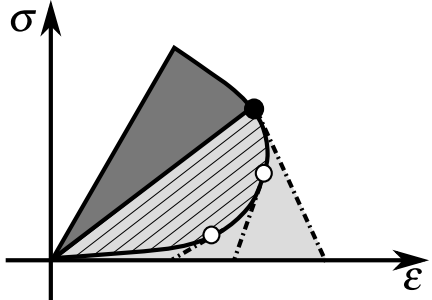

(a)

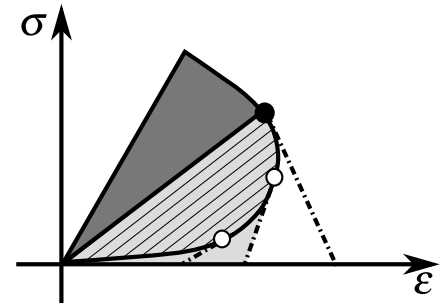

(b)

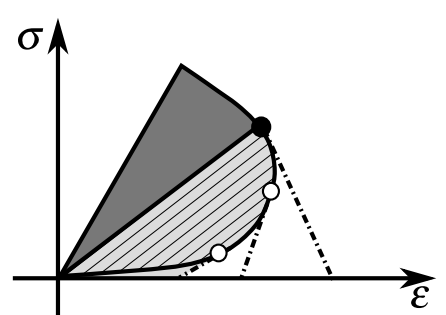

(c)

Figure 15. (a) If the energy to be transferred is estimated by means of the tangent line to $\sigma(\varepsilon)$ at model switching (black circle), a worse approximation is obtained than (b) if the tangent to $\sigma(\varepsilon)$ with some more load steps (white circle) is used. (c) The more load steps, the more accurate estimation of the energy not yet dissipated is obtained.

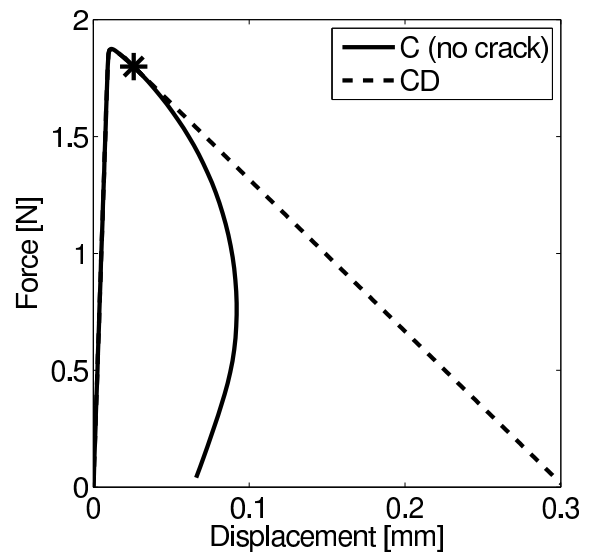

(a)

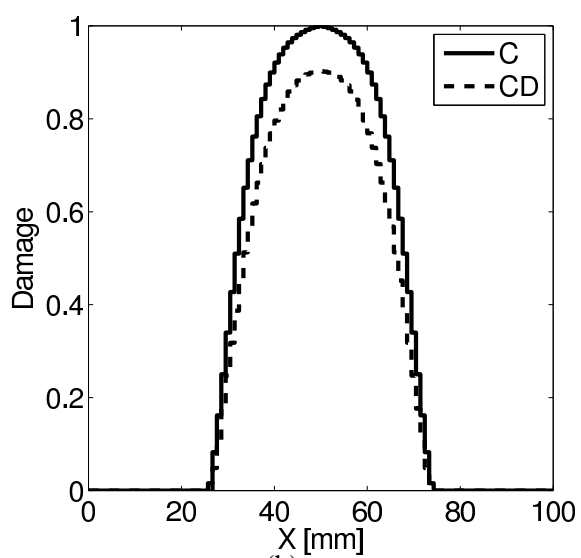

(b)

Figure 16. Uniaxial tension test (continuous and continuous-discontinuous approaches with a non-local damage model): (a) force displacement curves; (b) damage profiles.

\subsection{Energy balance for a multidimensional problem}

The extension of the proposed energy balance to a multidimensional setting is discussed in this section by means of a two-dimensional three-point bending test, see Figure 18 


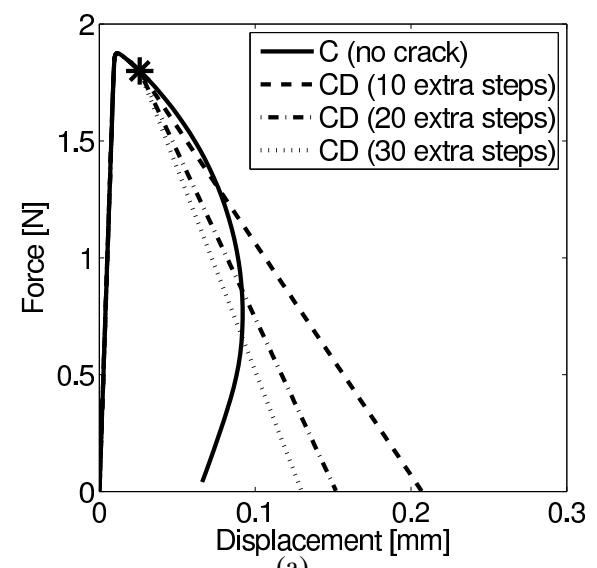

(a)

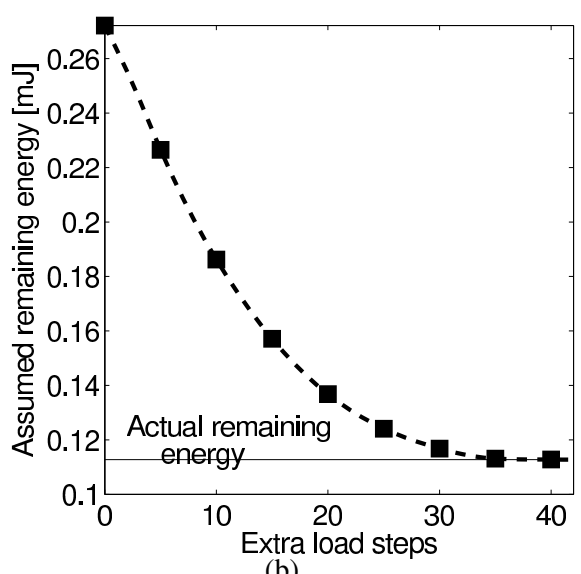

(b)

Figure 17. The more extra load steps are carried out with the continuous approach, the more accurate the energy to be transferred is estimated.

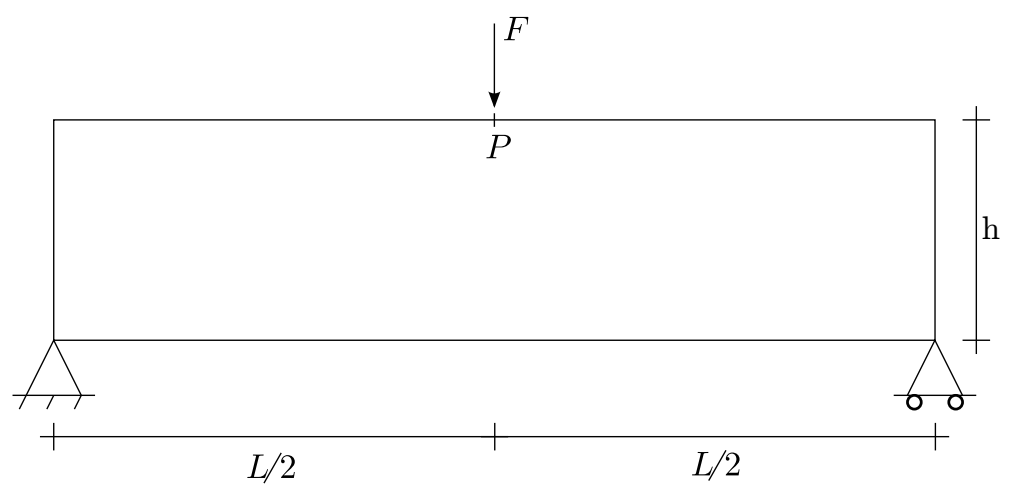

Figure 18. Three-point bending test: problem statement.

Here, the simplified Mazars damage model,

$$
Y=\sqrt{\sum_{i=1}^{3}\left(\max \left(0, \varepsilon_{i}\right)\right)^{2}}
$$

with $\varepsilon_{i}(i=1,2,3)$ the principal strains, and a bilinear damage evolution law, see Equation (13), are considered. A uniform mesh of $1640(41 \times 40)$ bilinear quadrilateral elements is used and the geometric and material parameters are summarised in Table IV

To begin with, a continuous simulation is carried out. A characteristic length $\ell=1 \mathrm{~mm}$ is considered. Results are shown in Figure 19 .

Let us now consider that as soon as damage reaches a critical value $D_{\text {crit }}=0.995$, a cohesive crack is introduced in that element and the proposed combined strategy is used. Due to the mode I behaviour of the three-point bending test, the traction-separation law

$$
\overline{\mathbf{t}}_{d}=\left\{\begin{array}{c}
\bar{t}_{n} \\
\bar{t}_{s}
\end{array}\right\}=\mathbf{T}\left\{\begin{array}{l}
\llbracket \boldsymbol{u} \rrbracket_{n} \\
\llbracket \boldsymbol{u} \rrbracket_{s}
\end{array}\right\}+\left\{\begin{array}{c}
t_{\text {crit }} \\
0
\end{array}\right\}=\left(\begin{array}{cc}
T & 0 \\
0 & 0
\end{array}\right)\left\{\begin{array}{l}
\llbracket \boldsymbol{u} \rrbracket_{n} \\
\llbracket \boldsymbol{u} \rrbracket_{s}
\end{array}\right\}+\left\{\begin{array}{c}
t_{\text {crit }} \\
0
\end{array}\right\}
$$

is employed, where $\llbracket \boldsymbol{u} \rrbracket_{n}$ and $\llbracket \boldsymbol{u} \rrbracket_{s}$ are the normal and sliding components of the crack opening $\llbracket \boldsymbol{u} \rrbracket$ respectively and $t_{\text {crit }}$ is the critical normal component of the traction vector.

This model switching - from the continuous to the combined strategy - entails one main consequence: damage in the cracked element is fixed to $D_{\text {crit }}$ and all points of this element start 
Table IV. Three-point bending test: geometrical and material parameters.

\begin{tabular}{lll}
\hline Meaning & Symbol & Value \\
\hline Length of the beam & $L$ & $300 \mathrm{~mm}$ \\
Height of the beam & $h$ & $100 \mathrm{~mm}$ \\
Young's modulus & $E$ & $30000 \mathrm{MPa}$ \\
Damage initiation state variable & $Y_{0}$ & $10^{-4}$ \\
Final state variable & $Y_{f}$ & $1.25 \times 10^{-2}$ \\
Poisson's ratio & $\nu$ & 0.00 \\
\hline
\end{tabular}

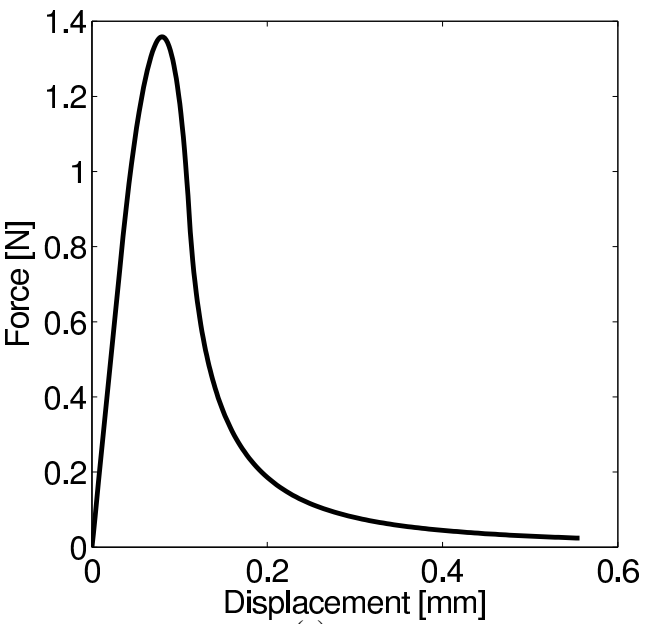

(a)

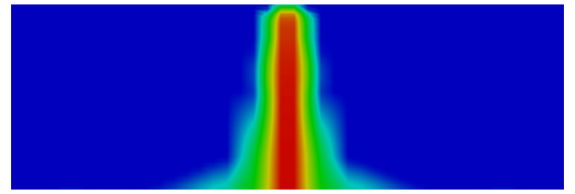

(b)

Figure 19. Three-point bending test (continuous approach): (a) force-displacement curve; (b) damage pattern.

to unload following the branch with slope $E\left(1-D_{\text {crit }}\right)$. In addition, points located near the crack faces unload following the secant branch with the stiffness at model switching.

Nevertheless, in contrast to the one-dimensional framework, ahead of the crack tip, damage keeps growing. In other words, the energy dissipated by the bulk with a combined technique is not the energy already dissipated in the bulk at model switching. This difference - between the one- and the multidimensional settings - is crucial when prescribing the energy balance. Indeed, in the uniaxial tension test discussed above, the energy balance is prescribed in all points of the bar, since all points unload secantly once the crack is introduced. However, if two- and three-dimensional problems are considered, the energy balance should be only prescribed in the area where points unload secantly.

In order to define this zone, that we have called crack influence zone, the following technique is used. First, the perpendicular to the direction of crack growth at the crack tip is considered. Then, the crack influence zone is defined as the area behind the crack tip, see Figure 20.

Once this area is defined, the energy balance presented by means of the uniaxial tension test can be used. That is, for each point in the crack influence zone, the energy not yet dissipated by the bulk is transferred to the crack. In order to estimate it, the tangent operator $\partial \sigma / \partial \varepsilon$ is computed via numerical differentiation, as in the one-dimensional case.

The results for the continuous and combined strategies are shown in Figure 21. In Figure 21(a), the force-displacement curves are seen. For comparison purposes, two different values of the cohesive parameter $T$ are considered. First, the energy balance proposed in [15] is employed. That is, an energy balance considering that all points unload following a softening branch is enforced. Second, our energy balance is prescribed. It is noted that considering the unloading behaviour of each 

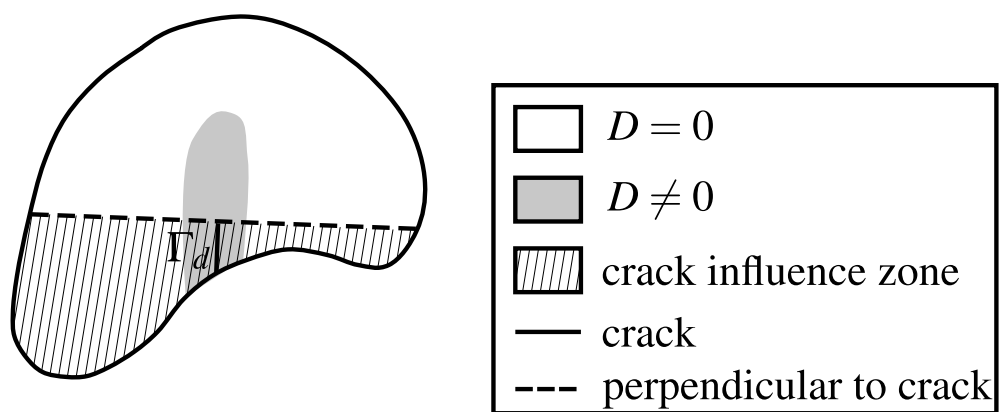

Figure 20. The perpendicular to the direction of crack growth allows to define the crack influence zone (striped area).

point in the crack influence zone - either softening or secant- the energy to be transferred is more accurately estimated. In Figures 21(b) 21(g), the obtained results - with the proposed energy balance - in terms of damage and deformation patterns (amplified by a factor of 100) are shown. Firstly, the continuous gradient-enhanced damage model with smoothed displacements is used, see Figures 21(b) 21(c). Then, as soon as damage reaches the critical damage value, a cohesive crack is introduced, see Figure 21(d) From that moment on, the crack propagates through a regularised damaged bulk, where damage keeps evolving ahead of the crack tip, see Figures 21(e) 21(g).

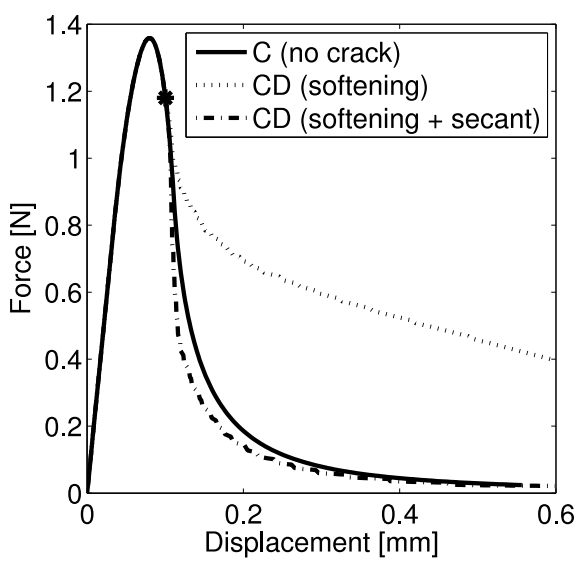

(a)

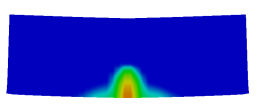

(b)

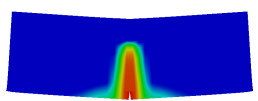

(d)

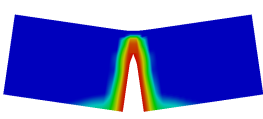

(f)

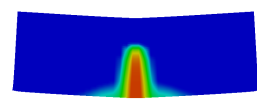

(c)

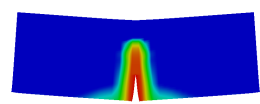

(e)

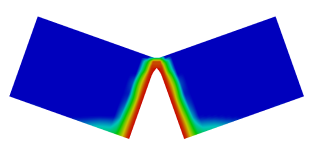

(g)

Figure 21. Three-point bending test (continuous and continuous-discontinuous approaches): (a) forcedisplacement curves; $(\mathrm{b})-(\mathrm{g})$ damage and deformed patterns $(\times 100)$ for increasing imposed displacements $u^{*}$.

As discussed, the main difference between the one- and the multidimensional settings is the domain where the energy balance is prescribed. Indeed, in 1D, the energy equilibrium is prescribed in all the damaged band. However, in 2D or 3D, the so-called crack influence zone must be defined. One means of avoiding the definition of this zone is to use a one-dimensional reference continuous simulation to extract the cohesive parameter $T$. In other words, as soon as the model switching is determined, an equivalent uniaxial tension test - that is, a uniaxial test with the same geometrical and material parameters of the multidimensional test - is carried out to compute the cohesive parameter $T$.

The capabilities of this new approximation are illustrated here by means of the previous threepoint bending test. Indeed, as soon as damage reaches a critical value $D_{\text {crit }}=0.995$, a cohesive crack, whose law is given by the softening parameter $T$ in Equation (23), is introduced. To compute the cohesive parameter, the one-dimensional energy balance discussed in Section 3.1 — with the 
material and geometrical parameters of the three-point bending test- is prescribed. Results are shown in Figure 22. As seen, little differences are observed if the energy balance is prescribed by means of the one-dimensional uniaxial test or with the two-dimensional setting. However, regarding the computational cost, this alternative way to define $T$ is very appealing.

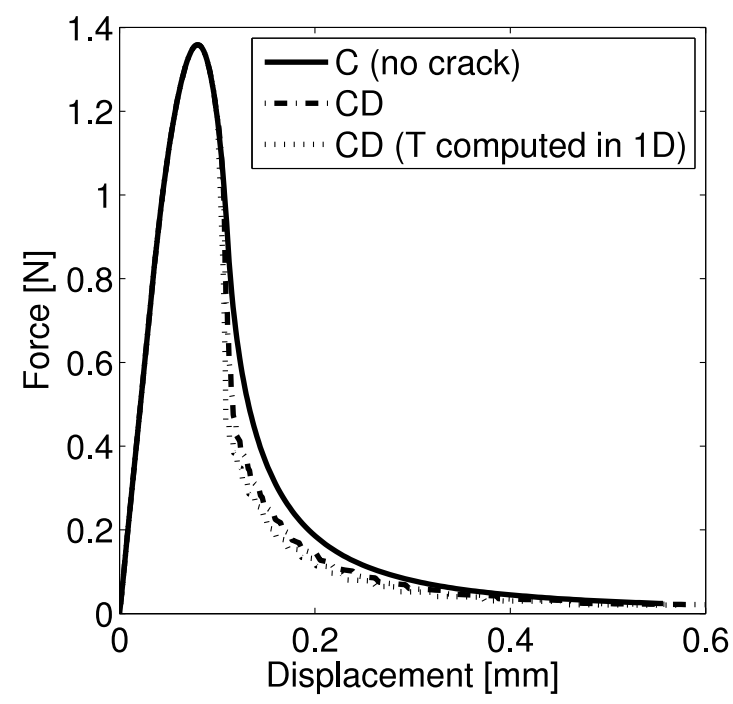

Figure 22. Three-point bending test (continuous and continuous-discontinuous approaches).

As a final remark, it is worth pointing out that the overall mechanical response and the crack opening are independent of the critical damage value, which is somewhat arbitrary, provided that the corresponding cohesive parameter $T$ is computed accurately. To show this, the three-point bending test is considered again. Now, the continuous-discontinuous strategy is activated later, for a critical value $D_{\text {crit }}=0.999$ (instead of $D_{\text {crit }}=0.995$ ). The results are shown in Figure 23 . With an accurate estimation of the energy to be transferred to the crack, two different values of $D_{\text {crit }}$ lead to two different values of $T$, which in turn result in very similar force-displacement and force-CMOD (crack-mouth opening displacement) curves, see the left column in Figure 23. On the contrary, a poor estimation of the cohesive law results in a different mechanical response and crack opening, see the right column in Figure 23.

\section{CONCLUDING REMARKS}

The continuous-discontinuous damage model presented in the companion paper [20] has been extended to cohesive cracks. That is, a non-local continuous formulation with smoothed displacements is combined with a propagating cohesive crack as soon as damage parameter equals or exceeds a critical value $D_{\text {crit }}<1$.

As suggested by the equivalent crack concept, the cohesive crack law is defined in such a way that the energy dissipated with a continuous damage model alone and with a continuous-discontinuous model are equal. The main difficulty of this energy balance is that after the switching - from the continuum to the discrete strategy - the continuous model is interrupted. Hence, without a reference continuous simulation, the energy to be transferred is not known and needs to be estimated.

Here, a new criterion to estimate this energy is proposed. The key idea of this new strategy consists in accounting for the different unloading branches of damage models — both secant and softening at switching. In other words, the unloading branch is approximated by means of the numerical derivative $\mathrm{d} \sigma(\varepsilon) / \mathrm{d} \varepsilon$ at switching. Compared to other existing techniques, where all points are considered to unload following the softening branch, here the energy that has to be transferred is more accurately estimated, as illustrated by means of two benchmark numerical tests. 
So far the proposed energy balance has been tested by means of a uniaxial tension test and a threepoint bending test. That is, only mode I loading conditions have been considered. Hence, it would be interesting to extend the proposed methodology to mode II and mode III loading conditions. Moreover, as discussed by means of the three-point bending tension test, carrying out a onedimensional reference continuous simulation to extract the cohesive parameter $T$ is an appealing way to compute the energetically equivalent cohesive crack law. Hence, it would be interesting to analyse the capabilities of this alternative way to define the cohesive slope in a more generalised setting.

\section{REFERENCES}

[1] Lemaitre J, Chaboche JL. Mechanics of solid materials. Cambridge University Press, 1990. ISBN-10: 0521328535.

[2] Jirásek M. Mathematical analysis of strain localization. Revue Européenne de Génie Civil 2007; 11(7-8):977-991, doi: 10.1080/177477120.2007.9692973.

[3] Jirásek M. Objective modeling of strain localization. Revue Française de Génie Civil 2002; 6(6):1119-1132, doi: 10.1080/12795119.2002.9692735

[4] Rabczuk T. Computational Methods for Fracture in Brittle and Quasi-Brittle Solids: State-ofthe-Art Review and Future Perspectives. ISRN Applied Mathematics 2013; 2013:38 pages, doi: $10.1155 / 2013 / 849231$

[5] de Borst R, Pamin J, Peerlings RHJ, Sluys LJ. On gradient-enhanced damage and plasticity models for failure in quasi-brittle and frictional materials. Computational Mechanics 1995; 17(1-2):130-141, doi: 10.1007/BF00356485

[6] Peerlings RHJ, de Borst R, Brekelmans WAM, Geers MGD. Gradient-enhanced damage modelling of concrete fracture. Mechanics of Cohesive-frictional Materials 1998; 3(4):323342, doi: 10.1002/(SICI)1099-1484(1998100)3:4<323::AID-CFM51>3.0.CO;2-Z.

[7] Hillerborg A, Modéer M, Petersson PE. Analysis of crack formation and crack growth in concrete by means of fracture mechanics and finite elements. Cement and Concrete Research 1976; 6(6):773-781, doi: 10.1016/0008-8846(76)90007-7

[8] Belytschko T, Black T. Elastic crack growth in finite elements with minimal remeshing. International Journal for Numerical Methods in Engineering 1999; 45(5):601-620, doi: 10.1002/(SICI)1097-0207(19990620)45:5<601::AID-NME598>3.0.CO;2-S

[9] Moës N, Dolbow J, Belytschko T. A finite element method for crack growth without remeshing. International Journal for Numerical Methods in Engineering 1999; 46(1):131-150, doi: 10.1002/(SICI)1097-0207(19990910)46:1<131::AID-NME726>3.0.CO;2-J

[10] Belytschko T, Gracie R, Ventura G. A review of extended/generalized finite element methods for material modeling. Modelling and Simulation in Materials Science and Engineering 2009; 17(4):043 001, doi: 10.1088/0965-0393/17/4/043001.

[11] Mazars J, Pijaudier-Cabot G. From damage to fracture mechanics and conversely: A combined approach. International Journal of Solids and Structures 1996; 33(20-22):3327-3342, doi: 10.1016/0020-7683(96)00015-7

[12] Jirásek M, Zimmermann T. Embedded crack model. Part II: combination with smeared cracks. International Journal for Numerical Methods in Engineering 2001; 50(6):1291-1305, doi: 10.1002/1097-0207(20010228)50:6<1291::AID-NME12>3.0.CO;2-Q 
[13] Wells GN, Sluys LJ, de Borst R. Simulating the propagation of displacement discontinuities in a regularized strain-softening medium. International Journal for Numerical Methods in Engineering 2002; 53(5):1235-1256, doi: 10.1002/nme.375.

[14] Simone A, Wells GN, Sluys LJ. From continuous to discontinuous failure in a gradientenhanced continuum damage model. Computer Methods in Applied Mechanics and Engineering 2003; 192(41-42):4581-4607, doi: 10.1016/S0045-7825(03)00428-6.

[15] Comi C, Mariani S, Perego U. An extended FE strategy for transition from continuum damage to mode I cohesive crack propagation. International Journal for Numerical and Analytical Methods in Geomechanics 2007; 31(2):213-238, doi: 10.1002/nag.537.

[16] Cazes F, Coret M, Combescure A, Gravouil A. A thermodynamic method for the construction of a cohesive law from a nonlocal damage model. International Journal of Solids and Structures 2009; 46(6):1476-1490, doi: 10.1016/j.ijsolstr.2008.11.019.

[17] Seabra MRR, César de Sá JMA, Andrade FXC, Pires FMA. Continuous-discontinuous formulation for ductile fracture. International Journal of Material Forming 2011; 4(3):271281, doi: 10.1007/s12289-010-0991-x.

[18] Cazes F, Simatos A, Coret M, Combescure A. A cohesive zone model which is energetically equivalent to a gradient-enhanced coupled damage-plasticity model. European Journal of Mechanics - A/Solids 2010; 29(6):976-989, doi: doi:10.1016/j.euromechsol.2009.11.003.

[19] Tamayo-Mas E. Continuous-discontinuous modelling for quasi-brittle failure: propagating cracks in a regularised bulk. PhD Thesis, Universitat Politècnica de Catalunya 2013.

[20] Tamayo-Mas E, Rodríguez-Ferran A. A medial-axis-based model for propagating cracks in a regularised bulk. Submitted.

[21] Rodríguez-Ferran A, Morata I, Huerta A. A new damage model based on non-local displacements. International Journal for Numerical and Analytical Methods in Geomechanics 2005; 29(5):473-493, doi: 10.1002/nag.422.

[22] Rodríguez-Ferran A, Bennett T, Askes H, Tamayo-Mas E. A general framework for softening regularisation based on gradient elasticity. International Journal of Solids and Structures 2011; 48(9):1382-1394, doi: 10.1016/j.ijsolstr.2011.01.022.

[23] Tamayo-Mas E, Rodríguez-Ferran A. Condiciones de contorno en modelos de gradiente con desplazamientos suavizados. Revista Internacional de Métodos Numéricos para Cálculo y Diseño en Ingeniería 2012; 28(3):170-176, doi: 10.1016/j.rimni.2012.03.006

[24] Jirásek M, Marfia S. Nonlocal damage models: displacement-based formulations. Proceedings of EURO- C2006: Computational Modelling of Concrete Structures, 2006; 381-390.

[25] Krayani A, Pijaudier-Cabot G, Dufour F. Boundary effect on weight function in nonlocal damage model. Engineering Fracture Mechanics 2009; 76(14):2217-2231, doi: 10.1016/j.engfracmech.2009.07.007.

[26] Pijaudier-Cabot G, Dufour F. Non local damage model: boundary and evolving boundary effects. European Journal of Environmental and Civil Engineering 2010; 14(6-7):729-749, doi: $10.1080 / 19648189.2010 .9693260$

[27] Cuvilliez S, Feyel F, Lorentz E, Michel-Ponnelle S. A finite element approach coupling a continuous gradient damage model and a cohesive zone model within the framework of quasi-brittle failure. Computer Methods in Applied Mechanics and Engineering 2012; 237240(0):244-259, doi: 10.1016/j.cma.2012.04.019. 

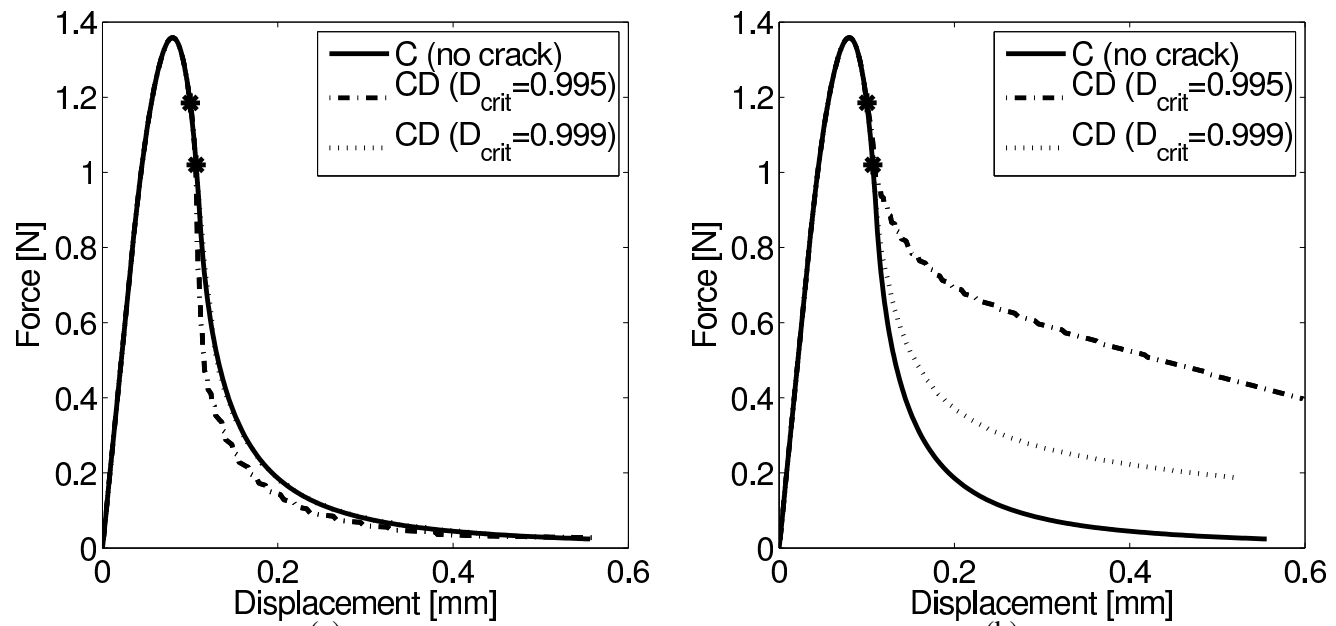

(a)
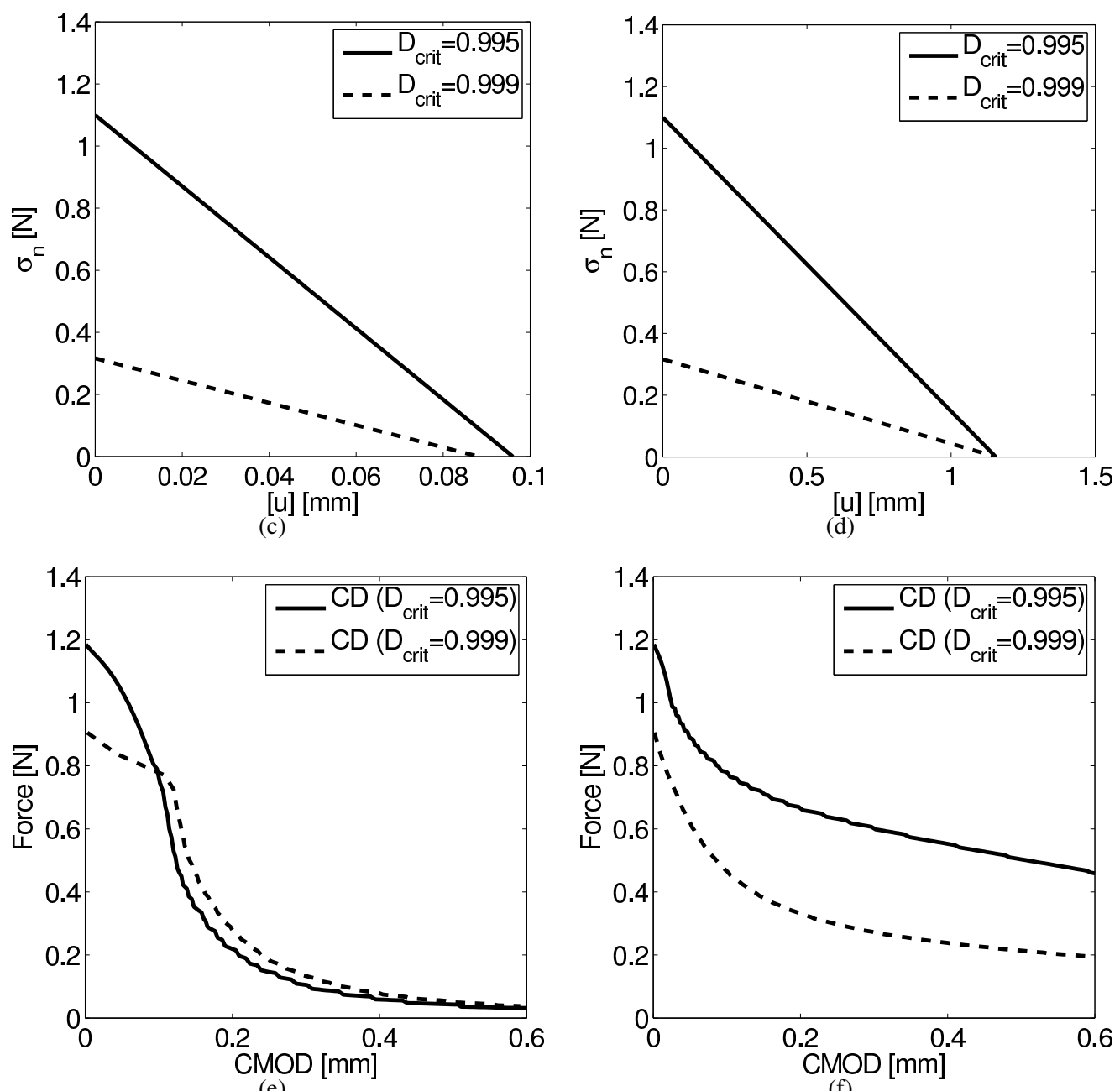

Figure 23. Three-point bending test (continuous and continuous-discontinuous approaches). Left column (results with the proposed energy balance): (a) force-displacement curves, (c) traction-separation law and (e) force-CMOD curves. Right column (results with the energy balance proposed in [15]): (b) forcedisplacement curves and (d) raction-separation law and (f) force-CMOD curves. 